Molecular Neurobiology

\title{
A novel Mecp2Y120D knockin model displays similar behavioral traits but distinct molecular features compared to the Mecp2-null mouse implying precision medicine for the treatment of Rett syndrome \\ --Manuscript Draft--
}

\begin{tabular}{|c|c|}
\hline Manuscript Number: & MOLN-D-18-00635R1 \\
\hline Article Type: & Original Article \\
\hline Keywords: & MeCP2; Rett syndrome; mouse models; chromatin accessibility; chromatin binding \\
\hline Corresponding Author: & $\begin{array}{l}\text { Nicoletta Landsberger, PhD } \\
\text { Universita degli Studi di Milano Facolta di Medicina e Chirurgia } \\
\text { ITALY }\end{array}$ \\
\hline First Author: & Anna Gandaglia \\
\hline \multirow[t]{19}{*}{ Order of Authors: } & Anna Gandaglia \\
\hline & Elena Brivio \\
\hline & Sara Carli \\
\hline & Michela Palmieri \\
\hline & Francesco Bedogni \\
\hline & Gilda Stefanelli \\
\hline & Anna Bergo \\
\hline & Barbara Leva \\
\hline & Chiara Cattaneo \\
\hline & Lara Pizzamiglio \\
\hline & Marco Cicerone \\
\hline & Veronica Bianchi \\
\hline & Charlotte Kilstrup-Nielsen, Ph.D \\
\hline & Ilda D'Annessa \\
\hline & Daniele Di Marino \\
\hline & Patrizia D'Adamo \\
\hline & Flavia Antonucci \\
\hline & Angelisa Frasca \\
\hline & Nicoletta Landsberger, Ph.D \\
\hline Abstract: & $\begin{array}{l}\text { MeCP2 is a fundamental protein associated with several neurological disorders, } \\
\text { including Rett syndrome. It is considered a multifunctional factor with a prominent role } \\
\text { in regulating chromatin structure; however, a full comprehension of the consequences } \\
\text { of its deficiency is still lacking. Here, we characterize a novel mouse model of Mecp2 } \\
\text { bearing the human mutation Y120D, which is localized in the methyl-binding domain. } \\
\text { As most models of Mecp2, the Mecp2Y120D mouse develops a severe Rett-like } \\
\text { phenotype. This mutation alters the interaction of the protein with chromatin but } \\
\text { surprisingly it also impairs its association with corepressors independently on the } \\
\text { involved interacting domains. These features, which become overt mainly in the } \\
\text { mature brain, cause a more accessible and transcriptionally active chromatin structure; } \\
\text { conversely, in the Mecp2-null brain we find a less accessible and transcriptionally } \\
\text { inactive chromatin. } \\
\text { By demonstrating that different MECP2 mutations can produce concordant } \\
\text { neurological phenotypes but discordant molecular features, we highlight the } \\
\text { importance of considering personalized approaches for the treatment of Rett }\end{array}$ \\
\hline
\end{tabular}


syndrome.

Powered by Editorial Manager $₫$ and ProduXion Manager $₫$ from Aries Systems Corporation 
A novel $M e c p 2^{Y 120 D}$ knockin model displays similar behavioral traits but distinct molecular features compared to the Mecp2-null mouse implying precision medicine for the treatment of Rett syndrome

Anna Gandaglia ${ }^{1}$, Elena Brivio ${ }^{1,6}$, Sara Carli $^{1}$, Michela Palmieri ${ }^{1}$, Francesco Bedogni ${ }^{1}$, Gilda Stefanelli ${ }^{1,7}$, Anna Bergo ${ }^{2}$, Barbara Leva ${ }^{2}$, Chiara Cattaneo ${ }^{1,8}$, Lara Pizzamiglio ${ }^{3}$, Marco Cicerone ${ }^{1}$, Veronica Bianchi ${ }^{1}$, Charlotte Kilstrup-Nielsen², Ilda D’Annessa ${ }^{4}$, Daniele Di Marino ${ }^{5}$, Patrizia D'Adamo $^{1}$, Flavia Antonucci ${ }^{3}$, Angelisa Frasca $^{3}$ and Nicoletta Landsberger ${ }^{1,3^{*}}$

${ }^{1}$ Neuroscience Division, IRCCS San Raffaele Scientific Institute, Milan, 20132, Italy

${ }^{2}$ Department of Biotechnology and Life Sciences, University of Insubria, Busto Arsizio (VA), 21052, Italy

${ }^{3}$ Department of Medical Biotechnology and Translational Medicine, University of Milan, Milan, 20100, Italy

${ }^{4}$ Istituto di Chimica del Riconoscimento Molecolare, CNR (ICRM-CNR), Milan, 20131, Italy

${ }^{5}$ Department of Informatics, Institute of Computational Science, Università della Svizzera Italiana, Lugano, 6900, Switzerland

${ }^{6}$ Present address: Department of Stress Neurobiology and Neurogenetics, Max Planck Institute of Psychiatry, International Max Planck Research School for Translational Psychiatry, Munich, 80804, Germany

${ }^{7}$ Present address: Department of Psychology, University of Toronto Mississauga, Mississauga, Ontario, L5L 1C6, Canada

${ }^{8}$ Present address: Department of Molecular Oncology and Immunology, the Netherlands Cancer Institute, Amsterdam, 1066 CX, The Netherlands

${ }^{*}$ Corresponding author. ORCID 0000-0003-0820-3155

Correspondence: nicoletta.landsberger@unimi.it; Tel.+390250330464.

\section{ACKNOWLEDGEMENTS}

We are extremely grateful to Giovanni Tonon and Simona Segalla (San Raffaele Scientific Institute) for their helpful technical suggestions. This work was mainly supported by the Italian parents' association "pro RETT ricerca" to N.L. The financial support of Fondazione Telethon - Italy [Grant no. GGP16015 to F.A.] is gratefully acknowledged. Functional experiments were supported by the Italian Ministry of Research and Education program "FIRB giovani” 2010, protocol number: RBFR10ZBYZ. Fondazione Veronesi (Milan, Italy) provided additional funding to F.B. Part of this work was carried out in 
ALEMBIC, an advanced microscopy laboratory established by IRCCS Ospedale San Raffaele and Università Vita-Salute San Raffaele.

\section{ABSTRACT}

$\mathrm{MeCP} 2$ is a fundamental protein associated with several neurological disorders, including Rett syndrome. It is considered a multifunctional factor with a prominent role in regulating chromatin structure; however, a full comprehension of the consequences of its deficiency is still lacking. Here, we characterize a novel mouse model of Mecp2 bearing the human mutation Y120D, which is localized in the methyl-binding domain. As most models of Mecp2, the Mecp2 $2^{Y 120 D}$ mouse develops a severe Rett-like phenotype. This mutation alters the interaction of the protein with chromatin but surprisingly it also impairs its association with corepressors independently on the involved interacting domains. These features, which become overt mainly in the mature brain, cause a more accessible and transcriptionally active chromatin structure; conversely, in the Mecp2-null brain we find a less accessible and transcriptionally inactive chromatin. By demonstrating that different $M E C P 2$ mutations can produce concordant neurological phenotypes but discordant molecular features, we highlight the importance of considering personalized approaches for the treatment of Rett syndrome.

\section{KEYWORDS}

MeCP2; Rett syndrome; mouse models; chromatin accessibility; chromatin binding 


\section{INTRODUCTION}

Mutations in the X-linked MECP2 (methyl-CpG-binding protein 2) gene cause a large spectrum of neurological disorders. Among these, Rett syndrome (RTT, OMIM \#312750) was the first identified and remains the most thoroughly characterized $M E C P 2$-related disorder [1, 2]. RTT is a pediatric neurodevelopmental disease that typically affects girls, as males fail to survive beyond the first few years of life. Hallmark of typical RTT is the regression of language and hand use after a period of apparently normal development that usually lasts 6-18 months. During and after this regression phase, patients develop a host of symptoms that typically include intellectual disability, respiratory abnormalities, hand stereotypies, ataxia, absence of speech, autonomic dysfunctions and epilepsy.

Although a comprehensive understanding of all its functions is still missing, MeCP2 is commonly defined as a ubiquitously expressed multifunctional protein mainly working as a transcriptional repressor. $\mathrm{MeCP} 2$, in fact, consists of two fundamental domains: a methyl-CpG binding domain (MBD) mediating its preferential binding to methylated DNA and a transcriptional repression domain (TRD) inhibiting gene expression through different mechanisms, including the recruitment of chromatin remodeling complexes to methylated DNA [3-6]. Moreover, in mature neurons, where MeCP2 is very abundant, the protein acts as an alternative linker histone, organizing a specialized chromatin structure that dampens transcriptional noise [7].

Even if most cases of RTT are caused by missense and nonsense mutations in $M E C P 2$, the first and so far most studied Mecp 2 model has been the full knockout mouse, generated and described in refs. [8, 9]. However, to mimic RTT more closely, few more animal models carrying human pathogenic mutations have been generated $[10,11]$.

Typically, Mecp2 mouse models recapitulate many RTT features. In particular, the hemizygous males appear grossly normal in the perinatal period but later they develop a progressive neurological phenotype whose onset and severity depend on the specific genetic mutation. These mice manifest locomotor deficits, hind limb clasping, hypotonia, reduced spontaneous movements, tremors, breathing defects and seizures [10]. While the complete absence of Mecp2 results in a premature death, generally occurring between 56 to 80 days of life $[8,9]$, the lifespan of knockin mice can vary from 95-110 days to more than 300 , according to the severity of the mutation [12].

Interestingly, several lines of evidence suggest that in null animals Mecp2 functions might be replaced by one or more other proteins, which therefore mask the consequences of its lack [7]. This suggests the 
importance of producing knockin models, that by ensuring the presence of the mutated protein, might provide better insights into MeCP2 functions [5, 10, 12-14]. For example, the $M e c p 2^{R 306 C}$ model was pivotal in revealing the recruitment of the NCoR/SMRT corepressor on DNA as a core MeCP2 function [5]. Similarly, Mecp $2^{T 158 M}$ and $M e c p 2^{R 133 C}$ mutations in mice were instrumental to determine the role of the MBD on protein stability and chromatin binding [12].

We have recently highlighted the relevance of Tyr-120 (Y120) residue for MeCP2 structure and function [15]. Its involvement in RTT was suggested by the identification of a patient carrying the pathogenic substitution of Y120 with aspartic acid (Y120D) [16]. The residue Y120 is located in the MBD of MeCP2 and it is highly conserved in mammals. In accordance with its location, we proved that the substitution of residue Y120 with a D impairs the ability of the MBD to interact with DNA [15]. Accordingly, previous studies demonstrated that an exogenously expressed MeCP2-Y120D derivative features a decreased affinity for heterochromatin $[17,18]$.

Based on these evidences and driven by the idea that specific mutations produce molecular consequences that might differ from the lack of Mecp2, we generated a novel mouse model carrying the human pathogenic mutation Y120D. We demonstrate that Mecp $2^{Y 120 D}$ mice show a harsh behavioral phenotype that is only slightly less severe than Mecp2-null mice. At the molecular level, the Y120D mutation impairs the ability of Mecp2 to acquire a tight binding to chromatin mainly in mature neurons and to recruit corepressors on heterochromatin. As a consequence, compared to the Mecp2-null model, the Y120D adult brain is characterized by a globally more open chromatin structure. Our data suggest that, in spite of overlapping RTT-like symptoms, different mouse models of Mecp2 might display largely different molecular phenotypes, a novel perspective for the field of Mecp2 studies. Furthermore, while 
our results reinforce the idea that Mecp2 functions change with brain maturation [7, 19], they challenge the general perception of the impact of Mecp2 mutations on neuronal chromatin architecture.

\section{MATERIALS AND METHODS}

\section{Plasmids}

Human eGFP-MeCP2-WT and its mutant derivatives eGFP-MeCP2-Y120D and -Y120F were obtained and described in [20]. The TBL1-mCherry construct [5] was kindly provided by Dr. Adrian Bird (Wellcome Trust Centre for Cell Biology, University of Edinburgh, United Kingdom).

\section{Animal husbandry}

Both mouse lines were housed in the animal facility of the San Raffaele Scientific Institute of Milan. Generation of the knockin (KI) mouse line is described in Supplementary Materials and Fig.S1. Mecp $2^{Y 120 D / y}$ males, heterozygous females and wild-type (WT) controls were obtained by crossing heterozygous females with WT males of either C57BL/6J or CD1 background purchased from Charles River. Genotyping was performed through PCR on genomic DNA purified from tail biopsies, using the following primers: common forward 5'-CAGGGCCTCAGAGACAAGC-3'; common reverse 5'GCAGATCGGCCAGACTTCC-3'; reverse for the KI allele 5'GGGTTAATTGATATCCAATTGGGATCC-3'. A 300 bp fragment is common for WT and knockin animals, while a $550 \mathrm{bp}$ fragment is specific for the mutated one. When needed (e.g. embryos for primary cell cultures or small pups), the animal sex was determined by using primers recognizing Jaridl (forward 5'-CCAGGATCTGACGACTTTCTACC-3', reverse 5'-TTCTCCGCAATGGGTCTGATT-3'), which produce a PCR fragment only in males.

Mating and genotyping of the CD1 Mecp2 $2^{\text {Bird }}$ mouse line are described in [21].

All procedures were performed in accordance with the European Community Council Directive 2010/63/UE for care and use of experimental animals; all the protocols were approved by the Italian Minister for Scientific Research and by the San Raffaele Scientific Institutional Animal Care and Use Committee in accordance with the Italian law.

\section{Phenotypic evaluation}

C57BL/6J or CD1 knockin mice and WT littermates were weekly tested for the presence or absence of RTT-like symptoms with a previously described scoring system [22]. During each session, mice were 
weighed and scored for their general condition, mobility, hind limb clasping and (only for CD1) tremor. Of note, the intermediate values of 0.5 and 1.5 were added to the canonical scores between 0 and 2 to provide a more detailed description of the phenotypic variability (for details see Supplementary Materials). All animals scoring 2 for general condition or tremor or rapidly losing weight were euthanized for ethical reasons. At the experimental endpoint, animals were euthanized through cervical dislocation after deep anesthesia with Avertin (Sigma-Aldrich, St Louis, MO, USA). At the time of death, brains were quickly removed, weighed and cortices dissected on ice. Day of euthanasia was considered as day of death without distinguishing it from natural death.

\section{Behavioral assessment}

Animals were maintained on an inverted $12 \mathrm{~h}$ light/darkness cycle at $22-24^{\circ} \mathrm{C}$.

Rotarod. Rotarod apparatus is composed by a horizontal rotating rod (diameter $3 \mathrm{~cm}$ ) on which mice, separated by large disks, have to keep their balance. The test was carried out in 2 days. Each day, 5 trials of $5 \mathrm{~min}$, separated by intervals of $30 \mathrm{~min}$, were presented to the mice. On day 1 , mice were placed on the apparatus with the rod rotating at $4 \mathrm{rpm}$ (rotations/minute) for $1 \mathrm{~min}$, then the rotation speed was increased every $30 \mathrm{sec}$ by $4 \mathrm{rpm}$. On day 2, mice were tested at a constant rotation speed corresponding to the average of the maximum speeds reached from all mice during day 1 . A trial ended when the mouse fell down or after $5 \mathrm{~min}$. The latency to fall down from the rod was taken as the dependent variable for every trial and was normalized for the mouse weight.

Grip. Grip test was performed with a horizontal metal bar (grip) strength dynamometer that mice grasp with the forelimb while suspended by their tail. Animals were gently pulled backward until they released the grip. The dependent variable was the maximal value obtained on a total of six trials.

Catwalk. Gait analysis was performed by using CatWalk XT System (Noldus Information Technology, Wageningen, the Netherlands, http://www.noldus.com). Mice were allowed to freely ambulate along an illuminated glass plate within a confined corridor $(50 \mathrm{x} 8 \mathrm{~cm})$ in a darkened room; footprints were recorded with a high-speed camera and subsequently analyzed with CatWalk XT 10.0 software (Noldus). Three trials/animal were analyzed and averaged to obtain stride length and base of support for front and hind right and left paws.

Spontaneous alternation. The test was performed in a dim illuminated room and mice were habituated to the environment for 1 hour before the test. The apparatus consisted in a cross maze with a central hub 
where the mouse was released and left free to explore for $10 \mathrm{~min}$. Number and sequence of entries in each arm were recorded throughout the test. An alternation was considered correct when no more than one repetition over five entries was made.

Trace Fear Conditioning. Auditory trace and delay fear conditioning were performed by placing the mice in an opaque conditioning chamber ( $\mathrm{x}$ X X H: $25 \times 17 \times 23 \mathrm{~cm}$; UgoBasile Instrument [Gemonio, Italy]) with a grid floor through which scrambled foot shocks could be delivered as unconditioned stimuli (US; $0.26 \mathrm{~mA}$ average intensity). The chamber was placed into a dimly lit $(<5 \mathrm{~lx})$ sound attenuating box (background noise level $55 \mathrm{~dB}$ ), and a speaker on top of the chamber allowed to deliver sounds as conditioning stimuli $(\mathrm{CS} ; 2000 \mathrm{~Hz})$.

All mice were pre-exposed to the test chamber for $10 \mathrm{~min}$ on the day before conditioning. Conditioning consisted in $1 \mathrm{~min}$ of baseline period (no stimulus) followed by 5 trials separated by $1 \mathrm{~min}$ of inter-trial intervals (ITI). In the trace fear conditioning, every trial is composed by the presentation of the CS (15 $\mathrm{sec}$ ) followed, $15 \mathrm{sec}$ later (trace), by the presentation of the shock for $2 \mathrm{sec}$. The freezing behavior was measured during the presentation of the CS. Twenty-four hours after fear conditioning, mice were placed again in the conditioning box, measuring their freezing behavior in the context test and tone test. Context testing consisted of 2 min without CS (“contextual freezing”); tone testing consisted of 1 min without CS followed by 1 min with the CS turned on in a new environment.

Data analysis. During Dark \& Light, Emergence and Novelty tests, animals were video-tracked using the EthoVision 2.3 system (Noldus Information Technology) with an image frequency of 4.2/sec. Raw data were transferred to Wintrack 2.4 for off-line analyses (http://www.dpwolfer.ch/wintrack). During Fear conditioning test, animals were continuously video-tracked using the ANY-maze system (Anymaze, Stoelting Co, Wood Dale, IL, USA, www.anymaze.com). The frequency of freezing (absence of movements except respiration) was continuously recorded. Statistical computations were done using Statview 5.0 (SAS Institute, Cary, NC, USA). Analysis of variance (ANOVA), alpha value 5\%, with factorial measures was used to test the statistical significance between genotypes effect.

\section{Primary cell cultures}

WT and Mecp2 $2^{Y 120 D / y}$ neurons were prepared from E15.5 or E17.5 cortices isolated from pregnant heterozygous females. Dissected tissues were washed in HBSS (Life-Technologies, Carlsbad, CA, USA), digested with $0.25 \%$ Trypsin-EDTA (Invitrogen, Carlsbad, CA, USA) and mechanically dissociated in 
dissecting medium (DMEM [Sigma-Aldrich], 10\% Horse serum [Euroclone, Pero, MI, Italy], 1\% glutamine [Sigma-Aldrich]). Cells were resuspended in neuronal culture medium (Neurobasal [Life Technologies], 2\% B-27 [Life Technologies], $1 \%$ glutamine) and plated on $0.1 \mathrm{mg} / \mathrm{ml}$ poly-L-lysine (Sigma-Aldrich) both on 6-multiwell plates at a density of 200000 cells/well and on 24-multiwell plates (containing glass coverslips) at a density of 20000 cells/well.

Mecp2-null mouse embryonic fibroblasts (MEF) were prepared from Mecp2 $2^{-/ y} \mathrm{CD} 1 \mathrm{E} 14.5$ embryos as previously described [20]. For DNA transfection, MEFs were plated on 12-multiwell plates on glass coverslips and transfected with lipofectamine 3000 (Invitrogen) as instructed by the manufacturer. Cells were analyzed 24 hours after transfection.

\section{Western Blot}

Whole brains and cortices harvested from $M e c p 2^{+/ y}$ and $M e c p 2^{Y I 20 D / y}$ mice were mechanically homogenized with a Potter-ELV glass grinder in lysis buffer $(50 \mathrm{mM}$ Tris- $\mathrm{HCl} \mathrm{pH} 7.4,500 \mathrm{mM} \mathrm{NaCl}$, 1\% Triton X-100, 2 mM EDTA, 1 mM DTT, 1X PhosSTOP [Roche, Basel, Switzerland], 1 X complete EDTA-free protease inhibitor cocktail [Roche]). Lysates were incubated $30 \mathrm{~min}$ on ice and sonicated (Amplitude $=100 \%$, three pulses for a total of $13 \mathrm{~min}$ ). After centrifugation at $13000 \mathrm{rpm}$ at $4^{\circ} \mathrm{C}$ for 15 min, supernatant was collected. Neurons from 6-multiwell plates were directly lysed in Laemmli buffer $6 \mathrm{X}$ and sonicated $(\mathrm{A}=100 \%$, for $13 \mathrm{~min})$. Proteins were electrophoretically separated on SDSpolyacrylamide gel and blotted on a nitrocellulose membrane using the Trans-Blot Turbo Transfer System from Bio-Rad (Hercules, CA, USA). Membranes were incubated 30 min in blocking solution (Tris-buffered saline containing 5\% nonfat milk and $0.1 \%$ Tween-20, pH 7.4) and then incubated with the primary antibody diluted in blocking solution overnight at $4^{\circ} \mathrm{C}$. The following primary antibodies were used: rabbit $\alpha$-MeCP2 (1:1000; Sigma-Aldrich), mouse $\alpha$-TuJ1 (1:10000; Sigma-Aldrich), rabbit $\alpha$-rpS6 (5G10, 1:1000; Cell Signaling Technology, Danvers, MA, USA), rabbit $\alpha$-P-rpS6 (Ser240/244, 1:1000; Cell Signaling Technology), rabbit $\alpha$-P-S164 (1:1000; Covance, Princeton, NJ, USA), rabbit $\alpha$ H1 (1:1000; GeneTex, Irvine, CA, USA). Secondary antibodies were either fluorescent (1:2000 AlexaFluor 488; Life Technologies) or HRP-conjugated (1:10000; Cell Signaling Technology). Signals were visualized and acquired using the fluorescence laser scanner Typhoon FLA 9000 or using the Chemiluminescence detection systems UVITEC ChemiDoc (Alliance 9.7, UVITEC Cambridge) or 
ChemiDoc Touch Imaging System (Biorad). Quantitative analyses were performed using either ImageJ software or ImageLab software from Bio-Rad.

\section{Immunofluorescence and histology}

Cells cultured on glass coverslips were washed with PBS and fixed in 4\% paraformaldehyde (PFA) for $20 \mathrm{~min}$ at room temperature (RT). Neurons were then permeabilized in $0.1 \%$ Tween-20 for $10 \mathrm{~min}$ at RT and blocked for 1 hour at RT (blocking solution: 10\% horse serum [Euroclone], $0.1 \%$ Triton X-100 in PBS), followed by an overnight incubation at $4^{\circ} \mathrm{C}$ with the rabbit $\alpha$-MeCP2 primary antibody $(1: 100$; Sigma-Aldrich) diluted in blocking solution. Cells were incubated with fluorescent secondary antibody (AlexaFluor $488 \alpha$-rabbit, 1:500 in blocking solution) for 1 hour at RT. Finally, DNA was counterstained with DAPI (1:1000 in PBS; Invitrogen) for $10 \mathrm{~min}$ at RT and coverslips were mounted with Prolong Gold reagent (Invitrogen).

Transfected MEFs were stained with DAPI (10 min at RT) after fixation. Images were acquired using Nikon microscope, objectives and software.

Mice were transcardially perfused with 4\% PFA. Brains were harvested, cryoprotected in sucrose $30 \%$, embedded in Tissue-Tek O.C.T. Compound (Sakura Finetek, Alphen aan den Rijn, The Netherlands) and cut in $12 \mu \mathrm{m}$ sections. Sections were washed thoroughly in PBS, antigen retrieved in $10 \mathrm{mM}$ sodium citrate, washed in $0.01 \%$ Tween-20 and incubated in $0.1 \mathrm{M}$ glycine. Tissues were blocked in blocking solution and incubated with antibodies as above (primary antibody: $\alpha$-ATRX H-300 1:100; Santa Cruz Biotechnology, Dallas, TX, USA. Secondary antibody: AlexaFluor $568 \alpha$-rabbit, 1:500). After counterstaining with DAPI, sections were mounted with Fluoromount-G (eBioscience, San Diego, CA, USA). Images were acquired using a Leica SP5 confocal microscope.

RNA Pol II-S5P staining was performed on nuclei isolated from $M e c p 2^{+/ y}, M e c p 2^{Y 120 D / y}$ and $M e c p 2^{-/ y}$ cortices at P40 (as described beneath in the Chromatin accessibility assay section). In detail, nuclei were permeabilized in PBST (0.01\% Triton X-100 in PBS), fixed in 4\% PFA and spotted on slides prior staining. Nuclei were then blocked for 1 hour at room temperature (RT) and incubated overnight at $4^{\circ} \mathrm{C}$ with $\alpha$-RNA Polymerase II-S5P (1:100, Abcam, ab5131). After washing in PBS, nuclei were incubated with AlexaFluor $488 \alpha$-rabbit (1:500; Life Technologies) for 2 hours at RT, counterstained with DAPI for $10 \mathrm{~min}$ at RT and finally mounted with Fluoromount-G (eBioscience). All images were acquired 
using a Leica SP5 confocal microscope and the RNA Polymerase II-SP5 activity was evaluated by measuring the intensity of the nuclear $\alpha$-RNA Polymerase II-S5P signal using ImageJ Software.

\section{Salt extraction assay}

Mecp2 chromatin affinity was assessed in brain tissues by performing salt extraction assays as described in refs. [3, 19].

\section{RNA purification, cDNA synthesis and qPCR}

Total RNA was extracted from $M e c p 2^{+/ y}$ and $M e c p 2^{Y 120 D / y}$ cortices using the RNeasy Mini Kit (QIAGEN, Hilden, Germany) following the manufacturer's instructions. RNA quality was assessed on $1 \%$ agarose gel. Total RNA was retro-transcribed using the RT2 First Strand Kit (QIAGEN). qPCRs were performed using SYBR Select Master Mix (Applied Biosystems, Foster City, CA, USA) and the following primers: Mecp2:

- Forward: 5' AAACCACCTAAGAAGCCCAAATC 3'

- Reverse: 5' TTGACAACAAGTTTCCCAGGG 3'

$18 S$ :

- Forward: 5' GTAACCCGTTGAACCCCATT 3'

- Reverse: 5' CCATCCAATCGGTAGTAGCG 3'

All samples were evaluated in triplicate and $18 S$ was used as normalizer.

Calcium imaging in cultured neurons

Cortical neurons at days in vitro (DIV) 4 or 13 were loaded with $5 \mathrm{mM}$ of the ratiometric $\mathrm{Ca}^{2+}$ dye Fura2 pentacetoxymethylester (Sigma-Aldrich) dissolved directly in neuronal medium solution for 35-40 min at $37^{\circ} \mathrm{C}$, washed and transferred to the recording chamber of an inverted microscope (Axiovert 100; Zeiss, Oberkochen, Germany) equipped with a calcium imaging unit [23]. Fluorescence images were obtained by alternatively illuminating cells at $345 \mathrm{~nm}$ and $380 \mathrm{~nm}$ wavelength and images ( $345 \mathrm{~nm}$ or $380 \mathrm{~nm}$ ) were acquired at $1 \mathrm{~Hz}$ frequency. After baseline acquisition, neurons were stimulated with 100 $\mu \mathrm{M}$ NMDA (Tocris) followed by $50 \mathrm{mM} \mathrm{KCl}$. Temporal $\mathrm{Ca}^{2+}$ intensity profiles (expressed as $\Delta \mathrm{F} 340 / 380$ fluorescence ratio) were calculated in discrete areas of interest from image sequences. Changes over baseline in the $\Delta \mathrm{F} 340 / 380$ fluorescence ratio higher than "100" were considered "calcium peak". To induce an appropriate NMDA stimulation, experiments were conducted in $\mathrm{Mg}^{2+}$-free Krebs-Ringer solution (KRH, in order to remove the $\mathrm{Mg}^{2+}$ block from the receptor) with tetrodotoxin $1 \mathrm{mM}$ (TTX), 
while the $\mathrm{KCl}$-induced depolarization has been reached by using normal $\mathrm{KRH}$ with $1 \mu \mathrm{M}$ TTX. Calcium transients were analyzed in about 150-200 cells per experimental group (as specified in legends). Nine glasses per group (1 field/glass) were analyzed at each developmental point.

\section{Chromatin accessibility assay}

To maintain nuclei intact, $M e c p 2^{+/ y}, M e c p 2^{Y I 20 D / y}$ and $M e c p 2^{-/ y}$ brains were mechanically homogenized on ice with Potter-ELV glass grinder in buffer A ( $0.25 \mathrm{M}$ sucrose, $60 \mathrm{mM} \mathrm{KCl}, 15 \mathrm{mM} \mathrm{NaCl}, 10 \mathrm{mM}$ MES pH 6.5, $5 \mathrm{mM} \mathrm{MgCl}_{2}, 1 \mathrm{mM} \mathrm{CaCl} 2,0.5 \%$ Triton X-100, $1 \mathrm{X}$ PhosSTOP [Roche], $1 \mathrm{X}$ complete EDTA-free protease inhibitor cocktail [Roche]). Samples were incubated on ice for $10 \mathrm{~min}$ and centrifuged at $7000 \mathrm{rpm}$ at $4{ }^{\circ} \mathrm{C}$ for $10 \mathrm{~min}$. Supernatants were discarded and nuclei were washed with buffer A. Nuclear pellets were resuspended in the digestion buffer B $(50 \mathrm{mM} \mathrm{NaCl}, 10 \mathrm{mM}$ Pipes pH 6.8, $5 \mathrm{mM} \mathrm{MgCl}_{2}, 1 \mathrm{mM} \mathrm{CaCl}_{2}$, 1X PhosSTOP, $1 \mathrm{X}$ complete EDTA-free protease inhibitor cocktail). Fifty $\mu 1$ of each sample were collected, supplemented with $3 \mu 1$ of $0.5 \mathrm{M}$ EDTA and conserved as the undigested fractions. The remaining volume was pre-warmed at $37^{\circ} \mathrm{C}$ and supplemented with 2.5 Units of MNase (Worthington Biochemical Corporation, Lakewood, NJ, USA) per 10 million of nuclei. Digested fractions were collected at the indicated time points. MNase digestion was stopped by adding $3 \mu \mathrm{l}$ of $0.5 \mathrm{M}$ EDTA to each fraction. DNA was phenol/chloroform extracted and quantified. For each fraction, $10 \mu \mathrm{g}$ of DNA were loaded on a $\% \mathrm{EtBr}$ agarose gel, which was run for approximately 5 hours. For each time point, ImageJ was used to obtain a densitometry plot and the amount of mononuclesomal DNA normalized on total DNA was plotted to highlight differences in the digestion rate between experimental groups.

\section{DAPI staining of MNase-treated nuclei}

Fresh isolated nuclei were obtained as above. Samples were resuspended in $300 \mu 1$ of buffer B and 2.5 Units $/ 50 \mu 1$ of $\mathrm{MNase}$ were added. Reactions were performed at $37^{\circ} \mathrm{C}$ and stopped at 0,5 , and $10 \mathrm{~min}$ by adding $3 \mu \mathrm{l}$ of $0.5 \mathrm{M}$ EDTA. Nuclei were then fixed in $4 \%$ PFA for $5 \mathrm{~min}$, spotted on a glass coverslip and stained with DAPI for $10 \mathrm{~min}$ at RT. Nuclei were mounted with Fluoromount-G and imaged using a 
Leica SP5 confocal microscope. DAPI intensity and heterochromatin area were calculated by using ImageJ software.

\section{5-hmC DNA ELISA assay}

Brain cortices were harvested from Mecp2 $2^{+/ y}, M e c p 2^{Y 120 D / y}$ and $M e c p 2^{-/ y} \mathrm{P} 40$ mice. Genomic DNA was extracted from $\leq 25 \mathrm{mg}$ of frozen brain cortices by using the Quick-DNA ${ }^{\mathrm{TM}}$ Miniprep Plus Kit (Zymo Research, Irvine, CA, USA) according to the manufacturer's instructions, with the exception that tissues were digested overnight with proteinase $\mathrm{K}$. The percentage of 5-hmC in $100 \mathrm{ng}$ of single-stranded DNA was quantified with the Quest 5hmC'т DNA ELISA Kit (Zymo Research), following the manufacturer's instructions. HRP color was allowed to develop for $30 \mathrm{~min}$ before reading plates at $415 \mathrm{~nm}$ with the Microplate Reader Model 680 (Bio-Rad).

\section{RESULTS}

$M e c p 2^{Y 120 D}$ knockin mice manifest a severe RTT-like phenotype and short lifespan

The mouse line carrying the human Y120D mutation was generated on a C57BL/6J background $\left(\right.$ Mecp $2^{Y 120 D}$ mice; Fig.1a; see Supplementary Materials and Fig.S1 for a detailed description of the procedures). Sequencing of hemizygous mutant mice, screened by PCR, proved the presence of the expected mutation (Fig.1b).

While the colony was maintained on a $\mathrm{C} 57 \mathrm{BL} / 6 \mathrm{~J}$ background, the mutant allele was also transferred on a CD1 (ICR) genetic background; the advantages of the CD1 background in Mecp2 studies are described in ref. [21]. Colonies were maintained by crossing heterozygous females to wild-type (WT) males, since hemizygous males were unable to induce pregnancy. To be noticed, the generated mutation does not affect Mecp2 mRNA levels (Fig.1c).

The severity of the Mecp2-Y120D mutation was evaluated using a well-established panel of parameters which include lifespan and phenotypic score [22]. As depicted in Fig.1d, hemizygous Mecp $2^{Y 120 D}$ males died prematurely (average lifespan of 97 and 121 days in the C57BL/6J and CD1 background, respectively). After weaning, mutated males became progressively symptomatic, as indicated by the increased phenotypic score (Fig.1e; Supplementary Materials and Fig.S2). On the other hand, heterozygous CD1 Mecp $2^{Y 120 D}$ females started showing typical RTT-like features only at 9 weeks of age (Fig.1f; Supplementary Materials and Fig.S2) and they did not manifest any apparent reduced longevity in the first year of life. $\mathrm{C} 57 \mathrm{BL} / 6 \mathrm{~J}$ heterozygous females were not analyzed in this study because in a time 
frame of over two years we did not succeed in generating a sufficient number of animals. In both sexes, similarly to null animals [21], tremor was the first symptom to appear along with worsening of the general conditions, while hind limb clasping and altered mobility appeared later and were characterized by higher variability (Supplementary Materials and Fig.S2). Body weight of Mecp2 animal models generally depends on mutation, sex and genetic background [21]; indeed, C57BL/6J Mecp2-null mice are notoriously underweight compared to WT, while the corresponding females are generally heavier. Compared to WT, Mecp2-null male animals on a CD1 background show no difference in body weight both at early and late postnatal time points. However, CD1 heterozygous females have a strong tendency towards obesity [21]. Our data show that while C57BL/6J Mecp $2^{Y 120 D}$ males were always lighter than WT littermates, CD1 mutated males showed a tendency to be lighter soon after birth, while becoming generally overweight in adulthood (Fig.1g). Conversely, the body weight of CD1 Mecp $2^{Y 120 D}$ heterozygous females was indistinguishable from WT littermates at early postnatal days, but it progressively increased with aging, leading to obesity (Fig.1h)

An early diagnostic criterion for Rett syndrome is deceleration of head growth, which often leads to microcephaly in infancy. Similarly, most of the Mecp2 animals generated so far (with the exception of the $M e c p 2^{308 X}$ mice [24]) are characterized by decreased brain size and weight. We weighted brains of pre- (P18) and symptomatic (P40 and P>130) CD1 Mecp2 $2^{Y I 20 D}$ males. Compared to WT, CD1 mutant brains exhibited an overall weight reduction of $12 \%, 13.5 \%$ and $17.5 \%$ at $\mathrm{P} 18, \mathrm{P} 40$ and $\mathrm{P}>130$, respectively; brains from symptomatic $\mathrm{C} 57 \mathrm{BL} / 6 \mathrm{~J}$ Mecp $2^{Y 120 D}$ males $(\mathrm{P}>80)$ had a $15.7 \%$ of weight reduction compared to WT brains (Fig. 1i).

The presence of RTT-like phenotypes was also analyzed through a battery of behavioral tests on CD1 Mecp $2^{Y 120 D}$ male mice and littermate controls. Results are summarized in Fig.2 and Table S1. Rotarod (Fig.2a), CatWalk (Fig.2b, c) and grip (Fig.2d) tests highlighted significant deficits in motor coordination, gait and anterior paws strength in juvenile (P30) and/or adult (P60) Mecp2 ${ }^{Y 120 D}$ mice.

We next examined learning ability of P30 and P60 Mecp $2^{Y 120 D}$ mice by performing the spontaneous alternation test. Working memory impairment was evident at P30, when knockin mice showed more than $30 \%$ reduction of correct responses with respect to controls, with no difference in the total number of visits (Fig.2e). P60 data have been omitted since the considerable decrease in spontaneous locomotor activity confounded the interpretation of results. We also found a deficit in associative memory through contextual and cued fear-conditioning tests (Fig.2f). Juvenile and adult knockin mice showed significant 
reduced freezing during the cue exposure $24 \mathrm{~h}$ after training, suggesting a specific impairment in the conditioned stimulus memory. By performing most of these tests also with CD1 Mecp2 null mice, we revealed that both Mecp2 lines manifest very similar behavioral performances (Fig.S3).

Anxiety-like and explorative behaviors were also assayed through different approaches (Supplementary Materials and Table S1) but no significant defect was observed. Notably, while anxiety is a common trait of RTT patients, in Mecp2 animal models this feature depends on the specific genetic mutation [25]. We completed the preliminary assessment of phenotypes typically evaluated in Mecp 2 animal models by testing whether knockin neurons exhibit functional defects similar to the Mecp2-null cells. We recently demonstrated that both in vivo and in vitro Mecp2-null neurons are characterized by a delayed maturation and impaired intrinsic responsiveness to external stimuli [26]. Thus, we cultured E15.5 Mecp2 $2^{Y I 2 O D}$ cortical neurons and probed neuronal responsiveness; the analyses were performed on immature (DIV4) or more mature (DIV13) neurons (Fig.2g). Calcium imaging analyses showed that the amplitude of KClinduced calcium transients is reduced in mutated neurons both at DIV4 and 13, confirming an impairment in the expression/sensitivity of voltage-operated calcium channels (VOCCs) and consequently a defective neuronal responsiveness to depolarization. Calcium imaging was also used to obtain information on the expression of functional NMDA receptors (Fig.2h). Whereas immature Mecp $2^{Y 120 D}$ neurons showed a significant reduction in NMDA-induced calcium peaks, mature neurons exhibited normal calcium transients associated with NMDA stimulation in spite of lower response to $\mathrm{KCl}$. These data confirm that Mecp2 mutations affect neuronal responsiveness; further, they suggest that Mecp2related phenotypes change along neuronal maturation.

\section{Y120D mutation affects Mecp2 abundance and chromatin binding mainly in mature neurons}

To gain insights into the molecular mechanisms responsible for the phenotypes affecting $M e c p 2^{Y 120 D}$ mice, we assessed Mecp2 levels and other molecular markers typically associated with Mecp2 functions. The results show that, while Mecp2-Y120D levels were normal in immature brains (E16, P6), they were significantly lower at P18 $(-56 \pm 4 \%, * * * \mathrm{P}<0.001$, Student's t test; Fig.3a) and $\mathrm{P} 40 \quad(-62 \pm 4 \%$, $* * * * \mathrm{P}<0.0001$, Student's t test; Fig.3a); a similar pattern was confirmed in in vitro cultured neurons (DIV7: $-29.7 \pm 12.3 \%$; DIV14: $-35.7 \pm 13.3 \%, * \mathrm{P}<0.05$, Student's t test; Fig. $3 b$ ).

In line with the Mecp2-null model, the mature knockin brain (P40) exhibited a reduction in rpS6 phosphorylation $\left(-43 \pm 4 \%,{ }^{*} \mathrm{P}<0.05\right.$, Student's t test; Fig.3c, upper panel), a hallmark of RTT progression $[27,28]$. Recently, we have demonstrated that phosphorylation of MeCP2 on Ser164 (S164), which is 
downregulated during neuronal maturation, globally diminishes MeCP2 binding to chromatin [19]. We thus assessed the levels of this phosphorylation in Mecp $2^{Y 120 D / y}$ adult mice, finding that it is significantly increased compared to WT $(+193 \pm 30 \%, * * * \mathrm{P}<0.001$, Student's t test; Fig. $3 \mathrm{c}$, lower panel).

Since a $50 \%$ reduction of protein levels in mice cannot explain the severity of the observed phenotype [29], we proceeded analyzing the consequences of Y120D mutation on Mecp2 activity. Previous studies demonstrated that the MeCP2-Y120D mutant exogenously expressed in non-neuronal cells shows a reduced accumulation on heterochromatic foci $[17,18]$. To confirm these results in cells expressing physiological levels of Mecp2, we compared the sub-nuclear localization of the WT and mutated proteins in cultured cortical neurons (Fig.3d). As expected, while the WT protein showed a clear accumulation on highly methylated heterochromatic foci starting from DIV7, Mecp2-Y120D remained mainly diffused in the nucleus along all stages of neuronal maturation, forming only few dots that do not overlap with DAPI-positive foci.

Our data thus suggest that the pathogenic mutation might directly and/or indirectly (through S164 phosphorylation; [19]) impairs Mecp2 affinity to chromatin, with the magnitude of the effect depending on brain maturation. Thus, through salt extraction experiments, we compared the affinity for chromatin of the WT and mutated protein in immature (P6) and mature (P40) mouse brains (Fig.3e-g). As reported [19], in mature brain WT Mecp2 is more tightly associated with chromatin compared to the immature brain. On the contrary, at $\mathrm{P} 40$ most of the mutant protein was extracted at $300 \mathrm{mM} \mathrm{NaCl}$, therefore failing to acquire a tighter association with chromatin (Fig.3f). To confirm this result with a complementary assay, an equal amount of brain nuclei isolated from WT and Mecp $2^{Y I 20 D}$ animals (P40) were incubated in buffers with increasing ionic strength and the amount of extracted Mecp2 analyzed by western blot (Fig.3g). As already demonstrated [3], WT Mecp2 was increasingly extracted at higher salt concentrations; indeed, the highest signal was observed at $1 \mathrm{M} \mathrm{NaCl}$, while almost no Mecp2 was detectable at $200 \mathrm{mM} \mathrm{NaCl}$. On the contrary, most of Mecp2-Y120D was extracted with a much lower concentration of $\mathrm{NaCl}(300-400 \mathrm{mM})$ with no further increase at $1 \mathrm{M} \mathrm{NaCl}$. Notably, the overall amount of the mutant protein was generally decreased in accordance with the protein levels depicted in Fig. $3 \mathrm{a}$. Although the affinity for chromatin of WT and mutated Mecp2 diverged more clearly in mature brain, 
quantification of three independent experiments revealed that at P6 Mecp2-Y120D is already less tightly bound to chromatin (Fig.3e).

Together, these data suggest that the Y120D mutation impairs both Mecp2 binding to chromatin and protein levels and that these phenotypes become progressively more evident when the WT MeCP2 acquires mature structural functions [7,19]

\section{$M e c p 2^{Y 120 D}$ and Mecp2-null brains exhibit different chromatin compaction}

Mecp2 is known to interact with different chromatin remodeling complexes and it is considered to play a major role in neuronal chromatin architecture $[4,30]$. We thus addressed whether Mecp2-Y120D maintains its ability to interact with relevant corepressors, such as NCoR and ATRX and whether this mutation affects chromatin compaction.

Mecp2-null MEFs were cotransfected with eGFP or eGFP-MeCP2 derivatives and TBL1-mCherry, and the ability of MeCP2 isoforms to recruit TBL1, part of the NCoR complex, on heterochromatin foci was assessed. In accordance with $[5,31]$, we demonstrated that no cell transfected with eGFP showed colocalization of TBL1-mCherry with heterochromatic foci (Fig.4a), whereas TBL1 was efficiently recruited on the DAPI-positive heterochromatic foci in $58 \%$ of cells expressing eGFP-MeCP2-WT and in $45 \%$ of cells expressing eGFP-MeCP2-Y120F. Importantly, only in 19\% of cells expressing MeCP2Y120D, TBL1-mCherry colocalized with the heterochromatic foci, suggesting that the interaction between Mecp2 and NCoR is significantly affected by the Y120D mutation. Of relevance, the presence of smaller and less bright green foci in cells that exogenously express eGFP-MeCP2-Y120D compared to cells transfected with eGFP-MeCP2-WT and eGFP-MeCP2-Y120F confirmed the impaired chromatin binding ability of the Y120D mutant.

Considering that the localization of the chromatin remodeling protein ATRX on pericentric heterochromatin of mouse neurons depends on its interaction with the MBD of Mecp2 [3, 6], we proceeded investigating and revealing a drastic change in ATRX localization in the Mecp2 $2^{Y I 20 D}$ brain with respect to controls (Fig.4b). Together these data confirm that the missense mutation Y120D alters the ability of MeCP2 to interact with and/or properly recruit on chromatin its corepressors, therefore affecting their sub-nuclear distribution. Given that such corepressors concur to chromatin landscaping, a time course of micrococcal nuclease (MNase) digestion of P6 and P40 brain nuclei was performed to analyze chromatin accessibility (Fig.5a and Fig.S4). Interestingly, while we were unable to detect any overt phenotype in the immature brain (Fig.S4), the MNase digestion pattern at P40 of the Mecp2 $2^{Y 120 D / y}$ 
sample showed a slightly faster accumulation of fragments corresponding to mononucleosomal DNA normalized on total DNA (Fig.5a). Quantification of three different experiments demonstrated a consistent trend that however did not reach the statistical significance (Fig.5b'). By digesting WT and Mecp2-null chromatin, the mutant sample appeared mildly less accessible; this tendency was confirmed by quantifying three independent experiments (Fig.5b"). Since these results might suggest that the complete absence of Mecp2 leads to compensatory mechanisms that differ from those generated by the presence of the mutated Mecp2, we corroborated these data with a complementary approach. Neuronal nuclei were thus purified from Mecp $2^{Y I 20 D / y}$ or Mecp2-null adult (P40) cortices and the corresponding WT control tissues, and heterochromatic and euchromatic accessibility was assessed by staining purified nuclei with DAPI at different time points of MNase digestion. By measuring both the stained area in heterochromatic foci (Fig.5c', left panel) and the average DAPI intensity (Fig.5c', left panel) in Y120D samples, we revealed that euchromatic and heterochromatic DNA is more rapidly digested in mutant nuclei, therefore suggesting a globally more accessible chromatin structure. Conversely, MNase treatment of Mecp2-null nuclei demonstrated that their heterochromatic DNA, but not bulk chromatin, is less digested than the control (Fig.5c', c', right panels). To verify whether this change in chromatin accessibility results in any functional alteration, transcriptional activity within single cortical nuclei was investigated by measuring the levels of phosphorylated RNA polymerase II (Pol II-S5P) in Mecp2 $2^{Y 120 D / y}$ or null P40 nuclei and their corresponding WT controls (Fig.5d; [32-35]). Signal specificity was confirmed by the lack of co-localization between DAPI foci (transcriptionally silent heterochromatin) and Pol II-S5P signal (transcriptionally active chromatin, Fig.5d). Importantly, a strong and significant increase of Pol II-S5P in Mecp $2^{Y 120 D / y}$ nuclei was observed $(+53.4 \pm 1.3 \%$, **** $\mathrm{P}<0.0001$, Student's t test; Fig.5d'), while the null nuclei showed a slight but significant reduction with respect to their controls ($22.9 \pm 0.9 \%, * * * * \mathrm{P}<0.0001$, Student's t test; Fig. $5 \mathrm{~d}$ '”).

Eventually, we reinforced these data analyzing whether WT, Mecp2-null and Mecp $2^{Y 120 D / y}$ mature brain cortices (P40) differ in their 5-hydromethylcytosine (5-hmC) content. In fact, a direct relationship between genome accessibility, transcriptional activity and 5-hmC levels has recently been proposed [36, 37]. In particular, methylated CG dinucleotides contained in highly compact chromatin are less frequently converted to 5-hmC, probably because of lower accessibility of Tet hydroxylases [36]. Fig.5e demonstrates that while $M e c p 2^{Y 120 D / y}$ cortices manifest a tendency to an increase in 5-hmC levels, Mecp2- 
null adult brains display a small but significant decrement., as already reported for Mecp2 deficient granule cells [38].

Overall these data support our hypothesis that the presence of Mecp2-Y120D diversely affects the general features of brain chromatin compared to the total absence of Mecp2.

\section{DISCUSSION}

The generation of several mouse models carrying different Mecp2 alterations proved instrumental to investigate $\mathrm{MeCP} 2$ functions and its involvement in Rett syndrome and MECP2 related disorders [10, 13]. Mecp2-null animals [8,9] were the first model generated and still represent the mostly used one. However, it has recently become evident that when Mecp2 is absent, its functions are replaced by compensatory mechanisms that might mask and confound the consequences of its loss [7]. These results highlighted the importance of producing other models with less severe genetic lesions, possibly mimicking human mutations $[5,10,12-14]$. Our manuscript describes the generation and the behavioral and molecular characterization of a novel knockin mouse model of Rett syndrome carrying the human missense mutation MECP2-Y120D, resulting in the substitution of tyrosine $120(\mathrm{Y})$ with an aspartic acid (D) [16]. Although the Y120 residue of MeCP2 has been linked to RTT only once so far, its involvement in MeCP2 affinity for heterochromatin has already been proved [17, 18]. Through molecular dynamics simulations, we have recently demonstrated that the Y120D mutation leads the methyl binding domain (MBD) of MeCP2 to sample an alternative conformational space that impairs its ability to interact with DNA. These results have been experimentally confirmed in electrophoretic mobility shift assays comparing the ability of WT and Y120D MBD polypeptides to bind methylated and unmethylated DNA [15]. Interestingly, the conversion of Y120 into F did not affect the DNA binding properties of the MBD of MeCP2. Similarly, here we demonstrate that WT and Y120F MeCP2 show a similar ability to recruit TBL1 on heterochromatin, while the mutant Y120D protein has lost such ability. All in all, these data suggest that harboring a tyrosine residue is not crucial for $\mathrm{MeCP} 2$ functions, while its conversion into aspartate, or possibly in a negatively charged residue, is a key detrimental step.

In line with the clinical features of the Y120D RTT patient [16], the Y120D mutation in mice leads to a harsh condition that is only slightly less severe compared to the total absence of the protein. Indeed, a progressive deterioration of the animal condition, in particular of the neurological aspects (mainly related 
to mobility, learning and memory functions), becomes overt after a short period of apparently normal development and eventually these mice die prematurely.

The molecular characterization of this novel mouse model revealed several features that are highly relevant for research on $\mathrm{MeCP} 2$ and Rett syndrome.

First, although the Y120D mutation is supposed to impair Mecp2 binding to DNA, the molecular phenotypes associated with the mutant protein (reduced affinity for chromatin, inability to accumulate on heterochromatic foci and bulk increase in chromatin accessibility) become evident mainly in mature neurons when protein levels are also halved. We recently suggested that Mecp2 changes its functions during brain maturation [19]. In particular, in developing brains and neurons the protein is not tightly bound to chromatin and largely co-sediments with nucleosome-free DNA. Possibly due to diverse posttranslational modifications (i.e. phosphorylation) during brain maturation, $\mathrm{MeCP} 2$ attains a tight association with nucleosomal DNA and chromatin, probably also acquiring structural functions, as already proposed [7]. Thus, it is conceivable that the molecular consequences of harboring the missense mutation Y120D become more severe in adult brain, when mutant MeCP2 fails in acquiring its expected structural functions. Nevertheless, the impaired DNA binding is expected to somehow affect the transcriptional properties of $\mathrm{MeCP} 2$ also in early development, therefore leading to functional alterations. Accordingly, we demonstrate that early responsiveness to stimuli of $M e c p 2^{Y I 20 D}$ cultured neurons is impaired.

Theoretically, all MeCP2 mutants characterized by a reduced DNA binding should share the herein described molecular phenotypes. Indeed, an effect on protein stability of the RTT mutations T158M and $\mathrm{R} 133 \mathrm{C}$ has already been proved. Interestingly, destabilization of mutant Mecp2 appeared to positively correlate with the reduction in DNA binding $[12,14]$. However, in apparent discordance with our results, the Mecp $2^{\text {TI58A }}$ mouse shows a significant decrement in proteins levels already at perinatal age that worsens with time [14]. Similarly, Lamonica et al. found that the protein expression levels of the mutant Mecp $2^{\mathrm{T} 158 \mathrm{M}}$ are reduced of almost $20 \%$ at $\mathrm{P} 0$ and further decreased at $\mathrm{P} 7$ and $\mathrm{P} 15$; no further decrement was observed beyond this developmental stage [39]

So far, it is not clear why mutations in T158 cause an earlier effect on protein levels compared to Y120D. We propose that unbound Mecp2 is more rapidly degraded in mature neurons than in immature cells, which are physiologically characterized by a more dynamic association of the protein with chromatin. In addition, as already suggested [40], Mecp2 degradation might be affected by its post-translational 
modifications and it is conceivable that Mecp2-Y120D is diversely modified with respect to T158A/M. Accordingly, we demonstrate that the Mecp $2^{Y 120 D / y}$ mature brain exhibits a significant increase in S164 phosphorylation. However, since the Y120D mutation somehow mimics the effect of Y120 phosphorylation that occurs more frequently in the immature brain [15], it is also possible that the Y120D missense mutation has a minor impact on the developing brain compared to the T158M/A mutations. Thus, although in the future we will investigate whether a different combination of post-translational modifications occurs on Mecp2-Y120D, currently we cannot exclude that the mutated Mecp2 T158M protein is more impaired in its DNA binding properties and therefore more effectively degraded in the brain since early development.

Several studies suggest that one of the most relevant activities of MeCP2 in brain is the recruitment of corepressors on methylated DNA, leading to an inaccessible and transcriptionally silent chromatin structure $[4,30]$. Considering that in mature neurons the protein is so abundant to bind the majority of methylated cytosines, its effects are likely exerted on the whole genome (Fig.6, "WT"). We demonstrate that in cells expressing the Y120D mutant, the molecular partners of Mecp2, such as NCoR and ATRX, are not properly recruited on heterochromatin. As a result, in the $M e c p 2^{Y I 20 D}$ mature brain, chromatin is globally more accessible compared to WT brain, in line with the increased levels of S5 phosphorylated RNA Polymerase II and the concomitant increase in 5-hmC levels, suggesting an increased transcriptional activity (Fig.6, "Y120D”). Unexpectedly, the consequences of the total absence of Mecp2 are quite different and do not appear to cause the hypothesized general decrease in chromatin compaction [19]. Indeed, we found that mature Mecp2-null brains do not have a more accessible chromatin structure, but instead exhibit a reduced MNase accessibility particularly on heterochromatic DNA. Furthermore, Mecp2-null brain nuclei manifest reduced levels of actively transcribing RNA polymerase II and 5-hmC. Skene et al. proposed that in mature neurons $\mathrm{MeCP} 2$ acts as a chromatin architectural factor whose absence is compensated by the up-regulation of histone H1 [7] (Fig.6, "null”). Our data confirm the existence of compensatory mechanism(s) but also suggest that these compensations lead to a more packed chromatin structure. In possible accordance, several studies profiling transcription in Mecp2-null brains unexpectedly reported the down-regulation of the majority of differentially expressed genes [4, 27]; further, it has been suggested that histone $\mathrm{H} 1$ stabilizes a tighter chromatin structure compared to $\mathrm{MeCP} 2[41]$. 
These data highlight that although mutations of Mecp2 might produce largely concordant behavioral phenotypes in mice, they can diversely compromise Mecp2 functions therefore leading to discordant compensatory mechanisms and/or alterations in chromatin structure. Although future studies will reveal the extent to which these diversities affect transcriptional profiles, our results indicate that effective therapeutic strategies might differ depending on the specific genetic mutation. For example, by increasing the expression of Mecp2-T158M, Lamonica et al. demonstrated that the reduced levels of the mutant protein are contributing to RTT pathogenesis [39]. Considering that mutations in the MBD might generally destabilize $\mathrm{MeCP} 2$, the authors proposed that therapies able to stabilize or increase the expression of mutant MeCP2 might have the potential to benefit most RTT patients carrying mutation in the MBD of MeCP2. However, we propose that the stabilization therapy might not be so beneficial in patients carrying a mutation that impairs the fundamental interaction of MeCP2 with NCoR [42], as it occurs with the MeCP2-Y120D alteration.

The molecular mechanism by which Y120D mutation impairs the NCoR binding is possibly due to a general change in the motions/dynamical profile of the MBD, while the T158M mutation mainly affects the structure of the domain [14]. Indeed, we demonstrated that, despite Y120 is not in direct contact with DNA, its mutation in D leads to an altered mobility of the domain and to a defective communication between the domain residues [15]. This impaired communication and profile of mobility/flexibility can be propagated to the entire protein, thus affecting the interaction with NCoR/SMRT. This long-range communication between the two protein domains could explain why the T158M and Y120D mutations have a different effect on the protein function although they are both located in the MBD.

To conclude, we suggest that only a thorough comprehension of the molecular alterations that affect Mecp2-null and knockin animal lines mimicking human pathogenic mutations will truly highlight the molecular consequences of MeCP2 deficiency and the possible existence of convergent molecular defects. Emphasis should be given on comparing Mecp2-null models with animals carrying human mutations that affect or not affect MeCP2 binding to DNA together (or not) with an influence on its ability to associate with its interactors. These comparisons will help clarifying whether treatment of $M E C P 2$-related diseases should consider a personalized approach in which patients are stratified in subgroups based on the molecular consequences of their genetic lesion. 


\section{AUTHOR CONTRIBUTIONS}

A.G and N.L conceptualized and designed most of the study. P.D-A and F.A. assisted the design and realization of the behavioral and electrophysiological studies, respectively. A.G., E.B., A.B., B.L., C.C., S.C., G.S., L.P., V.B., F.B., M.P., M.C. conducted the experiments, prepared most of the figures and revised the manuscript. A.F. helped with statistical analysis of data. N.L. wrote the manuscript with the assistance of A.F. D.D-M, I.D., C.K-N assisted in interpreting and discussing results.

\section{COMPLIANCE WITH ETHICAL STANDARDS}

Research involving animals was performed in accordance with the European Community Council Directive 2010/63/UE for care and use of experimental animals; all the protocols were approved by the Italian Minister for Scientific Research and by the San Raffaele Scientific Institutional Animal Care and Use Committee in accordance with the Italian law

\section{CONFLICTS OF INTERESTS}

The authors declare that they have not conflict of interest.

\section{REFERENCES}

1. Amir RE, Van den Veyver IB, Wan M, et al (1999) Rett syndrome is caused by mutations in Xlinked MECP2, encoding methyl-CpG-binding protein 2. Nat Genet 23:185-188. https://doi.org/10.1038/13810

2. Neul JL, Kaufmann WE, Glaze DG, et al (2010) Rett syndrome: Revised diagnostic criteria and nomenclature. Ann Neurol 68:944-950. https://doi.org/10.1002/ana.22124

3. Baker SA, Chen L, Wilkins AD, et al (2013) An AT-Hook Domain in MeCP2 Determines the Clinical Course of Rett Syndrome and Related Disorders. Cell 152:984-996. https://doi.org/10.1016/j.cell.2013.01.038

4. Bedogni F, Rossi RL, Galli F, et al (2014) Rett syndrome and the urge of novel approaches to study MeCP2 functions and mechanisms of action. Neurosci Biobehav Rev 46:187-201. https://doi.org/10.1016/j.neubiorev.2014.01.011

5. Lyst MJ, Ekiert R, Ebert DH, et al (2013) Rett syndrome mutations abolish the interaction of MeCP2 with the NCoR/SMRT co-repressor. Nat Neurosci 16:898-902. https://doi.org/10.1038/nn.3434

6. Nan X, Hou J, Maclean A, et al (2007) Interaction between chromatin proteins MECP2 and 
ATRX is disrupted by mutations that cause inherited mental retardation. Proc Natl Acad Sci 104:2709-2714. https://doi.org/10.1073/pnas.0608056104

7. Skene PJ, Illingworth RS, Webb S, et al (2010) Neuronal MeCP2 Is Expressed at Near HistoneOctamer Levels and Globally Alters the Chromatin State. Mol Cell 37:457-468. https://doi.org/10.1016/j.molcel.2010.01.030

8. Chen RZ, Akbarian S, Tudor M, Jaenisch R (2001) Deficiency of methyl-CpG binding protein2 in CNS neurons results in a Rett-like phenotype in mice. Nat Genet 27:327-331. https://doi.org/10.1038/85906

9. Guy J, Hendrich B, Holmes M, et al (2001) A mouse Mecp2-null mutation causes neurological symptoms that mimic Rett syndrome. Nat Genet 27:322-326. https://doi.org/10.1038/85899

10. Ricceri L, De Filippis B, Laviola G (2008) Mouse models of Rett syndrome: from behavioural phenotyping to preclinical evaluation of new therapeutic approaches. Behav Pharmacol 19:501517. https://doi.org/10.1097/FBP.0b013e32830c3645

11. Stearns NA, Schaevitz LR, Bowling H, et al (2007) Behavioral and anatomical abnormalities in Mecp2 mutant mice: A model for Rett syndrome. Neuroscience 146:907-921. https://doi.org/10.1016/j.neuroscience.2007.02.009

12. Brown K, Selfridge J, Lagger S, et al (2016) The molecular basis of variable phenotypic severity among common missense mutations causing Rett syndrome. Hum Mol Genet 25:558-570. https://doi.org/10.1093/hmg/ddv496

13. Bellini E, Pavesi G, Barbiero I, et al (2014) MeCP2 post-translational modifications: a mechanism to control its involvement in synaptic plasticity and homeostasis? Front Cell Neurosci 8:236. https://doi.org/10.3389/fncel.2014.00236

14. Goffin D, Allen M, Zhang L, et al (2012) Rett syndrome mutation MeCP2 T158A disrupts DNA binding, protein stability and ERP responses. Nat Neurosci 15:274-283. https://doi.org/10.1038/nn.2997

15. D'Annessa I, Gandaglia A, Brivio E, et al (2018) Tyr120Asp mutation alters domain flexibility and dynamics of MeCP2 DNA binding domain leading to impaired DNA interaction: Atomistic characterization of a Rett syndrome causing mutation. Biochim Biophys Acta - Gen Subj 1862:1180-1189. https://doi.org/10.1016/j.bbagen.2018.02.005

16. Inui K, Akagi M, Ono J, et al (2001) Mutational analysis of MECP2 in Japanese patients with atypical Rett syndrome. Brain Dev 23:212-215. https://doi.org/10.1016/S0387-7604(01)001978

17. Agarwal N, Becker A, Jost KL, et al (2011) MeCP2 Rett mutations affect large scale chromatin 
organization. Hum Mol Genet 20:4187-95. https://doi.org/10.1093/hmg/ddr346

18. Kudo S, Nomura Y, Segawa M, et al (2003) Heterogeneity in residual function of MeCP2 carrying missense mutations in the methyl CpG binding domain. J Med Genet 40:487-493. https://doi.org/10.1136/jmg.40.7.487

19. Stefanelli G, Gandaglia A, Costa M, et al (2016) Brain phosphorylation of MeCP2 at serine 164 is developmentally regulated and globally alters its chromatin association. Sci Rep 6:28295. https://doi.org/10.1038/srep28295

20. Bergo A, Strollo M, Gai M, et al (2015) Methyl-CpG Binding Protein 2 (MeCP2) Localizes at the Centrosome and Is Required for Proper Mitotic Spindle Organization. J Biol Chem 290:3223-3237. https://doi.org/10.1074/jbc.M114.608125

21. Cobolli Gigli C, Scaramuzza L, Gandaglia A, et al (2016) MeCP2 Related Studies Benefit from the Use of CD1 as Genetic Background. PLoS One 11:e0153473. https://doi.org/10.1371/journal.pone.0153473

22. Guy J, Gan J, Selfridge J, et al (2007) Reversal of neurological defects in a mouse model of Rett syndrome. Science (80- ) 315:1143-7. https://doi.org/10.1126/science.1138389

23. Antonucci F, Corradini I, Morini R, et al (2013) Reduced SNAP-25 alters short-term plasticity at developing glutamatergic synapses. EMBO Rep 14:645-651. https://doi.org/10.1038/embor.2013.75

24. Shahbazian MD, Young JI, Yuva-Paylor LA, et al (2002) Mice with truncated MeCP2 recapitulate many Rett syndrome features and display hyperacetylation of histone H3. Neuron 35:243-254. https://doi.org/10.1016/S0896-6273(02)00768-7

25. Katz DM, Berger-Sweeney JE, Eubanks JH, et al (2012) Preclinical research in Rett syndrome: setting the foundation for translational success. Dis Model Mech 5:733-745. https://doi.org/10.1242/dmm.011007

26. Bedogni F, Cobolli Gigli C, Pozzi D, et al (2016) Defects During Mecp2 Null Embryonic Cortex Development Precede the Onset of Overt Neurological Symptoms. Cereb Cortex 26:2517-2529. https://doi.org/10.1093/cercor/bhv078

27. Li Y, Wang H, Muffat J, et al (2013) Global Transcriptional and Translational Repression in Human-Embryonic-Stem-Cell-Derived Rett Syndrome Neurons. Cell Stem Cell 13:446-458. https://doi.org/10.1016/j.stem.2013.09.001

28. Ricciardi S, Boggio EM, Grosso S, et al (2011) Reduced AKT/mTOR signaling and protein synthesis dysregulation in a Rett syndrome animal model. Hum Mol Genet 20:1182-1196. 
https://doi.org/10.1093/hmg/ddq563

29. Samaco RC, Fryer JD, Ren J, et al (2008) A partial loss of function allele of Methyl-CpG-binding protein 2 predicts a human neurodevelopmental syndrome. Hum Mol Genet 17:1718-1727. https://doi.org/10.1093/hmg/ddn062

30. Guy J, Cheval H, Selfridge J, Bird A (2011) The Role of MeCP2 in the Brain. Annu Rev Cell Dev Biol 27:631-652. https://doi.org/10.1146/annurev-cellbio-092910-15412

31. Kruusvee V, Lyst MJ, Taylor C, et al (2017) Structure of the MeCP2-TBLR1 complex reveals a molecular basis for Rett syndrome and related disorders. Proc Natl Acad Sci 114:E3243E3250. https://doi.org/10.1073/pnas.1700731114

32. Egloff S, Murphy S (2008) Role of the C-terminal domain of RNA polymerase II in expression of small nuclear RNA genes. Biochem Soc Trans 36:537-539. https://doi.org/10.1042/BST0360537

33. Hirose Y, Ohkuma Y (2007) Phosphorylation of the C-terminal Domain of RNA Polymerase II Plays Central Roles in the Integrated Events of Eucaryotic Gene Expression. J Biochem 141:601-608. https://doi.org/10.1093/jb/mvm090

34. Linhoff MW, Garg SK, Mandel G (2015) A High-Resolution Imaging Approach to Investigate Chromatin Architecture in Complex Tissues. Cell 163:246-255. https://doi.org/10.1016/j.cell.2015.09.002

35. Phatnani HP, Greenleaf AL (2006) Phosphorylation and functions of the RNA polymerase II CTD. Genes Dev 20:2922-2936. https://doi.org/10.1101/gad.1477006

36. Lister R, Mukamel EA, Nery JR, et al (2013) Global Epigenomic Reconfiguration During Mammalian Brain Development. Science (80- ) 341:1237905-1237905. https://doi.org/10.1126/science.1237905

37. Ludwig AK, Zhang P, Hastert FD, et al (2017) Binding of MBD proteins to DNA blocks Tet1 function thereby modulating transcriptional noise. Nucleic Acids Res 45:2438-2457. https://doi.org/10.1093/nar/gkw1197

38. Mellén M, Ayata P, Dewell S, et al (2012) MeCP2 Binds to 5hmC Enriched within Active Genes and Accessible Chromatin in the Nervous System. Cell 151:1417-1430. https://doi.org/10.1016/j.cell.2012.11.022

39. Lamonica JM, Kwon DY, Goffin D, et al (2017) Elevating expression of MeCP2 T158M rescues DNA binding and Rett syndrome-like phenotypes. J Clin Invest 127:1889-1904. https://doi.org/10.1172/JCI90967

40. Thambirajah AA, Eubanks JH, Ausió J (2009) MeCP2 post-translational regulation through 
PEST domains: two novel hypotheses. BioEssays 31:561-569. https://doi.org/10.1002/bies.200800220

41. Riedmann C, Fondufe-Mittendorf YN (2016) Comparative analysis of linker histone H1, MeCP2, and HMGD1 on nucleosome stability and target site accessibility. Sci Rep 6:33186. https://doi.org/10.1038/srep33186

42. Tillotson R, Selfridge J, Koerner M V., et al (2017) Radically truncated MeCP2 rescues Rett syndrome-like neurological defects. Nature. https://doi.org/10.1038/nature24058

\section{FIGURE LEGENDS}

Fig.1. The novel Mecp2 $2^{Y 120 D}$ mouse line manifests severe RTT-like symptoms and short lifespan (a) Schematic representation of the Mecp2 locus targeting strategy exploited for the insertion of the Y120 mutation. See also Fig.S1. (b) DNA sequencing electropherogram showing the presence in the generated animals of the single point mutation $\mathrm{T}>\mathrm{G}$ - highlighted - that leads to the substitution of $\mathrm{Y} 120$ with an aspartic acid (D). (c) qPCR assay shows no difference in the Mecp2 mRNA levels of P30 C57BL/6J $M e c p 2^{Y 120 D / y}(\mathrm{n}=4)$ cortices, compared to $\mathrm{Mecp}^{+/ y}(\mathrm{n}=3)$. Data are represented relative to Mecp2 $2^{+/ y}$, as mean \pm s.e.m. Significance is calculated by Student's t test. (d) Mecp $2^{Y 120 D / y}$ mice in C57BL/6J and CD1 background ( $\mathrm{n}=8$ and 11 , respectively) show reduced lifespan compared to $M e c p 2^{+/ y}$ littermates ( $\mathrm{n}=6$ and 9, respectively). Significance is calculated by Log-rank (Mantel-Cox) test $\left({ }^{*} P<0.05, * * * * P<0.0001\right)$. (e) Plots showing the progression of the phenotypic score (cumulated for general condition, mobility, hind limb clasping and -only for CD1- tremor values) of C57BL/6J and CD1 mutated males (animals evaluated: $\mathrm{n}=6 \mathrm{C} 57 \mathrm{BL} / 6 \mathrm{~J}$ and $10 \mathrm{CD} 1 M e c p 2^{Y 120 D / y}$ vs $7 \mathrm{C} 57 \mathrm{BL} / 6 \mathrm{~J}$ and $\left.9 \mathrm{CD} 1 \mathrm{Mecp} 2^{+/ y}\right)$. Analyses ended when there were less than 3 animals per group. Data are represented as mean \pm s.e.m. Significance is calculated by two-way ANOVA. Plots of single variable are showed in Fig.S2. (f) Plot showing the progression of the phenotypic score (cumulated for general condition, mobility, hind limb clasping and tremor values) of CD1 heterozygous females (animals evaluated: $\mathrm{n}=7 \mathrm{Mecp} 2^{\mathrm{Yl} 20 \mathrm{D} /+}$ vs $8 \mathrm{Mecp}^{+/+}$). Analyses ended when there were less than 3 animals per group. Data are represented as mean \pm s.e.m. Significance is calculated by two-way ANOVA. Plots of single variable are showed in Fig.S2. (g) Plots showing the progression of total body weight of C57BL/6J and CD1 mutated males in comparison with control littermates (animals evaluated: $\mathrm{C} 57 \mathrm{BL} / 6 \mathrm{~J} \mathrm{n}=6 \mathrm{Mecp}^{Y 120 D / y}$ vs $7 \mathrm{Mecp}^{+/ y}$; CD1 pups $\mathrm{n}=19$ $M e c p 2^{Y 120 D / y}$ vs n=17 Mecp2 $2^{+/ y} ; \mathrm{CD} 1$ adult $\mathrm{n}=10 \mathrm{Mecp}^{Y 120 D / y}$ vs $\left.9 \mathrm{Mecp}^{+/ y}\right)$. Analyses ended when there were less than 3 animals per group. Data are represented as mean \pm s.e.m. Significance is calculated by multiple t test $(* P<0.05, * * P<0.01, * * * P<0.001, * * * * P<0.0001)$. (h) Plot showing the progression of 
total body weight of CD1 heterozygous females in comparison with control littermates (animals evaluated: pups $\mathrm{n}=18 \mathrm{Mecp}^{\mathrm{YI2OD} /+}$ vs $\mathrm{n}=14 \mathrm{Mecp}^{+/+}$; adult $\mathrm{n}=7 \mathrm{Mecp}^{\mathrm{YI2OD/+}}$ vs $\left.8 \mathrm{Mecp} 2^{+/+}\right)$. Analyses ended when there were less than 3 animals per group. Data are represented as mean \pm s.e.m. Significance is calculated by multiple $\mathrm{t}$ test $\left({ }^{*} P<0.05,{ }^{* *} P<0.01,{ }^{* * *} P<0.001, * * * * P<0.0001\right)$. (i) Brain weight was measured in juvenile (P18, $\mathrm{n}=4 M e c p 2^{Y 120 D / y}$ vs $\left.7 M e c p 2^{+/ y}\right)$, young adult $\left(\mathrm{P} 40, \mathrm{n}=18 \mathrm{Mecp} 2^{Y 120 D / y}\right.$ vs 14 Mecp $\left.^{+/ y}\right)$ and adult (CD1 P $>130 \mathrm{n}=4$ per genotype, C57BL/6J $\mathrm{P}>80 \mathrm{n}=5$ per genotype) mice. Measures of brain weights are expressed as mean \pm s.e.m, Significance is calculated by Student's $t$ test $(* P<0.05$, **P $P<0.01, * * * P<0.001, * * * * P<0.0001)$.

Fig.2. CD1 $M e c p 2^{Y 120 D}$ mice are impaired in motor performance and short-term memory. See also Table S1. (a) Rotarod test showing the persistence on the rotating bar of P30 Mecp2 $2^{Y I 20 D / y}(\mathrm{n}=24)$ compared to $M e c p 2^{+/ y}(\mathrm{n}=14)$ littermate mice. P30 Mecp $2^{Y 120 D / y}$ mice show altered motor coordination (acceleration phase, day 1) and increased muscle fatigue (constant speed, day 2). (b, c) Gait analyses with CatWalk XT software show small strides in the knockin mice. Analyses were performed on P30 $M e c p 2^{Y 120 D / y}(\mathrm{n}=24)$ and Mecp2 $2^{+/ y}(\mathrm{n}=14)$, and P60 Mecp2 $2^{Y 120 D / y}(\mathrm{n}=12)$ and $M e c p 2^{+/ y}(\mathrm{n}=16)$ littermate mice. Stride length of right front (RF), right hind (RH), left front (LF) and left hind (LH) paws were measured in (b) and the base length of front and hind paws in (c). (d) The strength of front paw muscles was measured through grip strength test on P30 Mecp2 $2^{Y I 20 D / y}(\mathrm{n}=24)$ and Mecp2 $2^{+/ y}(\mathrm{n}=14)$, and P60 $\operatorname{Mecp} 2^{Y 120 D / y}(\mathrm{n}=12)$ and $\operatorname{Mecp}^{+/ y}(\mathrm{n}=17)$ littermate mice. The panel shows maximum values. (e) Working memory defects in P30 Mecp $2^{Y 120 D / y}(\mathrm{n}=12)$ and $M e c p 2^{+/ y}(\mathrm{n}=17)$ littermate mice assessed with the spontaneous alternation test. Mecp $2^{Y 120 D / y}$ mice show decreased percentage of correct alternation with the same number of entries compared to WT animals. (f) Impaired tone (cue; conditioned stimulus CS) memory in P30 Mecp2 $2^{Y 120 D / y}(\mathrm{n}=13)$ and P60 Mecp2 $2^{Y 120 D / y}(\mathrm{n}=22)$ mice compared to their Mecp2 $2^{+/ y}$ littermates $(\mathrm{P} 30, \mathrm{n}=15 ; \mathrm{P} 60, \mathrm{n}=9)$ during the auditory fear conditioning test. Graphs represent the percentage of freezing during the 5 tones (CS) of training phase (left panels), during the context test 24 hours after training (middle panels) and during the cue test (right panels). Data are presented as means \pm s.e.m. For statistical analyses see Materials and Methods $\left({ }^{*} P<0.05, \quad * * P<0.01, \quad * * * P<0.001\right.$, $* * * * P<0.0001)$. (g) Calcium imaging experiments in cultured E15.5 cortical neurons reveal precocious and persistent functional alterations in $M e c p 2^{Y 120 D / y}$ neurons. Representative traces and relative quantification of $\mathrm{KCl}$-induced calcium transients in WT and Mecp2 $2^{Y 120 D / y}$ cells are depicted. Whereas resting calcium levels are indistinguishable in the two groups during neuronal development 
$\left(M e c p 2^{+/ y}=0.487 \pm 0.005\right.$ vs $M e c p 2^{Y 120 D / y}=0.480 \pm 0.006 ; \mathrm{t}$ test Mann-Whitney Rank Sum Test: $\left.P=0.373\right)$

$\mathrm{KCl}$ administration leads to reduced calcium influxes in both DIV4 (g': Mecp $2^{+/ y}=0.39 \pm 0.013$ vs $M e c p 2^{Y 120 D / y}=0.29 \pm 0.009$; t test Mann Whitney Rank Sum Test: $* * * P<0.001$; number of analyzed cells: $M e c p 2^{+/ y}=184$ vs Mecp $\left.2^{Y 120 D / y}=147\right)$ and DIV13 Mecp $2^{Y 120 D / y}$ cultures (g': Mecp2 $2^{+/ y}=0.404 \pm 0.010$ vs $M e c p 2^{Y 120 D / y}=0.368 \pm 0.009$; t test Mann-Whitney Rank Sum Test: ${ }^{*} P=0.005$; number of analyzed cells: $M e c p 2^{+/ y}=155$ vs $M e c p 2^{Y 120 D / y}=189$; resting calcium levels $M e c p 2^{+/ y}=0.50 \pm 0.004$ vs Mecp $2^{Y 120 D / y}=0.52 \pm 0.006$; $\mathrm{t}$ test Mann-Whitney Rank Sum Test $P=0.096$ ). (h) NMDA delivery induces smaller calcium transients in immature DIV4 mutated neurons (h': DIV4; Mecp $2^{+/ y}=0.313 \pm 0.011$ vs Mecp $2^{Y 120 D / y}=0.267 \pm 0.011 ; \mathrm{t}$ test: $\left.* P=0.027\right)$ and comparable calcium responses in mature cells $(\mathbf{h} "$ : DIV13; Mecp $2^{+/ y}=0.409 \pm 0.012$ vs $M e c p 2^{Y I 20 D / y}=0.408 \pm 0.012 ; \mathrm{t}$ test: $\mathrm{p}=0.612$ ). Number of analyzed cells: Mecp $^{+/ y}$ DIV4=150, DIV13=184; Mecp2 $2^{Y 120 D / y}$ DIV4=150, DIV13=189).

\section{Fig.3. The Y120D mutation affects Mecp2 abundance and its chromatin binding properties in} mature neurons. (a) Representative images and western blot quantification of Mecp2 protein levels assessed on E16 brains and P6, P18, P40 cortices of Mecp $2^{Y 120 D / y}$ and $M e c p 2^{+/ y} \mathrm{CD} 1$ males $(\mathrm{n} \geq 5$ per genotype at each time point). Data are represented relative to $M e c p 2^{+/ y}$, as mean \pm s.e.m. Significance is calculated by Student's t test $(* * * P<0.001, * * * * P<0.0001)$. (b) Representative images and western blot quantification of Mecp2 protein levels of Mecp2 $2^{Y 120 D / y}$ and Mecp2 ${ }^{+/ y}$ E15.5 cortical neurons at different DIVs ( $\mathrm{n} \geq 10$ per genotype at each time point). Data are represented relative to $M e c p 2^{+/ y}$, as mean \pm s.e.m. Significance is calculated by Student's t test $(* P<0.5)$. (c) Western blot quantification showing altered P-rpS6 and P-S164 levels in cortices of P40 Mecp2 $2^{Y 120 D / y} \mathrm{CD} 1$ mice (n=7 Mecp2 $2^{Y 120 D / y}$ vs 5 Mecp2 $2^{+/ y}$ ). Data are represented relative to $M e c p 2^{+/ y}$, as mean \pm s.e.m. Significance is calculated by Student's t test $\left({ }^{*} P<0.5, * * * P<0.001\right)$. (d) Representative images of $M e c p 2^{+/ y}$ and $M e c p 2^{Y 120 D / y}$ cultured neurons established from E17.5 cortices and immunostained at different DIV for DAPI (left panels) and Mecp2 (middle panels). Mecp2 $2^{Y I 20 D / y}$ nuclei show diffused staining that does not overlap with DAPIheterochromatic foci (white arrows). Images were taken at $100 \mathrm{X}$ magnification. (e, f) Upper panels: representative western blots showing salt extraction of WT or mutant Mecp2 with increasing concentrations of $\mathrm{NaCl}$ from $\mathrm{P} 6$ and $\mathrm{P} 40$ mouse $\mathrm{CD} 1$ brains. $\mathrm{H} 1$ was included as control of the salt extraction procedure. Lower panels: quantification of the fraction of WT and Y120D Mecp2 extracted with different concentration of $\mathrm{NaCl}$. Data are represented as mean \pm s.e.m. Significance is calculated by two-way ANOVA followed by Bonferroni post-hoc test. $(* P<0.05, * * P<0.01, \quad * * * P<0.001$, 
****P<0.0001). (g) Nuclei were purified from Mecp $2^{+/ y}$ and $M e c p 2^{Y 120 D / y} \mathrm{P} 40$ brains and resuspended in buffers containing $200 \mathrm{mM}, 300 \mathrm{mM}, 400 \mathrm{mM}$ or $1 \mathrm{M} \mathrm{NaCl}$. Extracted Mecp2 was tested by western blot $(n=2)$.

Fig.4. The Y120D mutation affects the capacity of Mecp2 to recruit its corepressors on chromatin.

(a) Representative images of Mecp2-null mouse embryonic fibroblasts (MEF) transfected with eGFPMeCP2 derivatives to study the recruitment of the TBL1-mCherry construct on heterochromatic foci [5]. TBL1 is efficiently recruited on the DAPI-positive heterochromatic foci in $58 \%$ of cells expressing eGFP-MeCP2-WT ( $n=60)$, in 45\% of cells expressing eGFP-MeCP2-Y120F ( $n=60)$ and only in 19\% of the eGFP-MeCP2-Y120D ones $(n=62)$. In no cell transfected with eGFP alone TBL1-mCherry $(n=20)$ was recruited on heterochromatic foci. Images were taken at $40 \mathrm{X}$ magnification. The contrast and brightness parameters for each channel were adjusted to obtain a clear representation of the results. (b) Representative images showing the heterochromatic localization of ATRX in WT brains, in contrast to the diffuse staining of ATRX observable in Y120D sections ( $\mathrm{n}=3$ animals per genotype; 60X magnification).

Fig.5. Mecp2 $2^{Y 120 D}$ brain exhibits different chromatin features compared to Mecp2-null and WT brains. (a) Representative images of chromatin accessibility assessed by digesting with MNase P40 $M e c p 2^{+/ y}$ and Mecp2 $2^{Y 120 D / y}$ and Mecp2 $2^{+/ y}$ and Mecp2 $2^{-/ y}$ brain nuclei. Purified digested DNA was loaded on a $2 \%$ agarose gel. For a similar analysis performed at P6, see Fig.S4. (b) Graphs showing the fraction of mononucleosomal DNA on total DNA at each time point $(5,10$ and $20 \mathrm{~min})$ of $M e c p 2^{+/ y}$ and $M e c p 2^{Y 120 D / y}\left(\mathbf{b}^{\prime}, \mathrm{n}=3\right)$ and $M e c p 2^{+/ y}$ and $M e c p 2^{-/ y}(\mathbf{b}$ ', $\mathrm{n}=3)$ samples. Data are expressed as mean \pm s.e.m. No statistical significance was reached by two-way ANOVA followed by Bonferroni post-hoc test. (c) DAPI staining of MNase-treated nuclei isolated from P40 Mecp2 $2^{+/ y}$ and $M e c p 2^{Y 120 D / y}$ and $M e c p 2^{+/ y}$ and $M e c p 2^{-/ y}$ littermate cortices. Purified nuclei were digested with MNase for 5 and $10 \mathrm{~min}$ before staining. Undigested nuclei (UT) were DAPI stained for comparison ( $\mathrm{n}=60$ to 110 nuclei for each experimental group). Heterochromatic area stained by DAPI was measured in Mecp2 $2^{Y I 20 D / y}$ nuclei and Mecp2-null nuclei (c'), while total nuclear intensity is displayed in $\mathbf{c}$ '. Data are expressed as percentage of the untreated WT and are represented as mean \pm s.e.m. Significance was calculated by two-way ANOVA followed by Bonferroni post-hoc test $(* * P<0.01, * * * P<0.001, * * * * P<0.0001)$. (d) Mecp $2^{Y I 20 D / y}$ nuclei show higher Pol II-S5P transcriptional signal compared to Mecp2 $2^{-/ y}$. Representative images of RNA Pol 
II S5P staining of Mecp $2^{Y I 20 D / y}$ and $M e c p 2^{-/ y}$ cortical nuclei at P40 and their respective controls are displayed. Graphs represent the intensity of Pol II-S5P signal of d') $M e c p 2^{+/ y}$ and Mecp2 $2^{Y 120 D / y}$ nuclei and d") $M e c p 2^{+/ y}$ and $M e c p 2^{-/ y}$ nuclei (n=100 nuclei for each experimental group). Data are expressed as percentage of WT and are represented as mean \pm s.e.m. Significance is calculated by Student's t test $(* * * * P<0.0001)$. (e) Graphs represent the percentage of 5 -hmC in brain cortices of $\mathrm{P} 40 \mathrm{Mecp} 2^{+/ y}$ $M e c p 2^{Y 120 D / y}$ and $M e c p 2^{-/ y}$ mice ( $\mathrm{n}=5$ for Mecp $2^{Y 120 D / y}$ and respective controls; $\mathrm{n}=5$ for $M e c p 2^{-/ y}$ and respective controls). Data are expressed as percentage of WT and are represented as mean \pm s.e.m. Significance is calculated by Student's t test $\left({ }^{*} P<0.5\right)$.

Fig.6. A model for the different chromatin structure in Mecp2-null and Mecp2 $2^{Y 120 D / y}$ brains. Several studies have proposed that in brain $\mathrm{MeCP} 2$ is globally bound to chromatin where it recruits different remodeling chromatin complexes, therefore leading to a specialized chromatin structure ("WT" panel). Loss of MeCP2 protein would lead to compensatory mechanisms that double histone H1 levels [7] and lead to a more condensed and transcriptionally inert chromatin structure ("null" panel). In the Y120D knockin brain, the labile interaction between $\mathrm{MeCP} 2$ and DNA and the concomitant delocalization of its corepressors such as NCoR and ATRX lead to a more accessible and transcriptionally active chromatin 

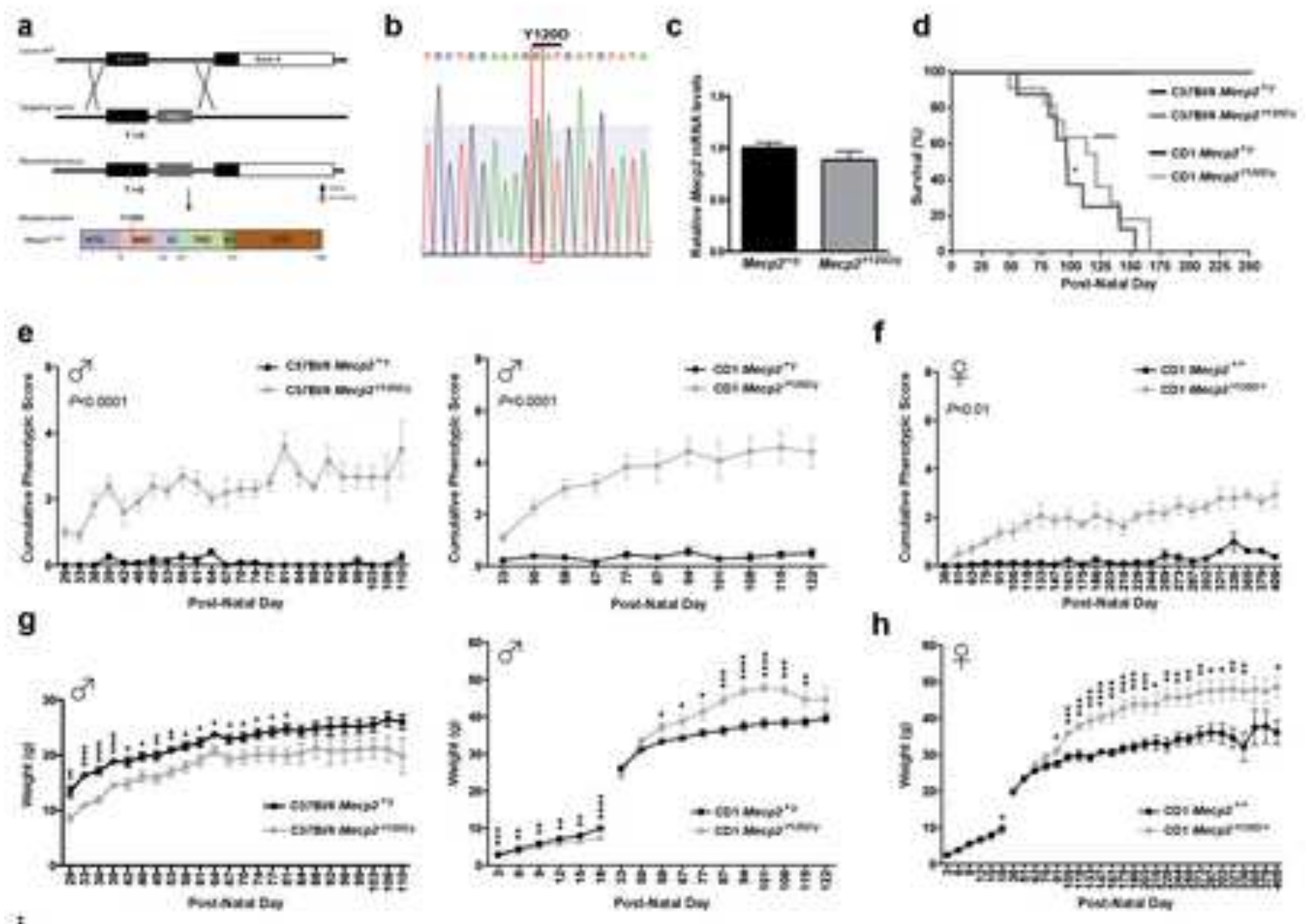

f

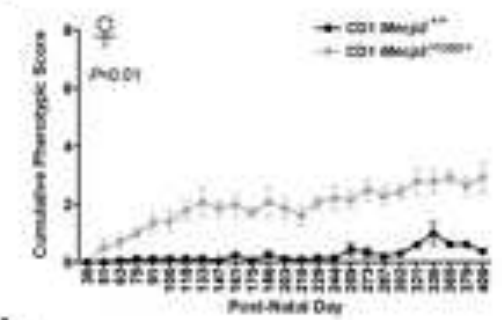

h

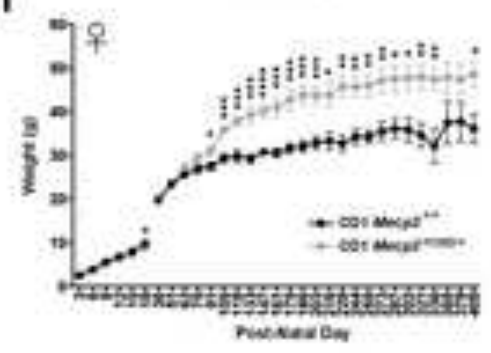

\begin{tabular}{|c|c|c|c|}
\hline \multicolumn{2}{|c|}{ Drain wetight } & Hropt" & Anplower \\
\hline \multirow{3}{*}{ Ceni } & min & emresos & $\begin{array}{c}0.0 .10,000 \\
t\end{array}$ \\
\hline & rat & buti ieseses & esmasont \\
\hline & Powe & batr, bears & Un tean, \\
\hline
\end{tabular}

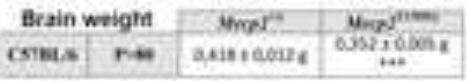


a

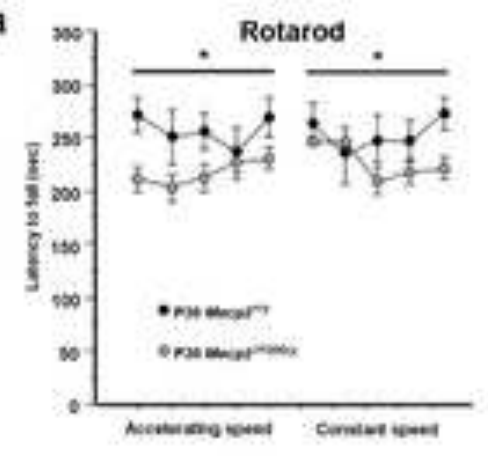

c

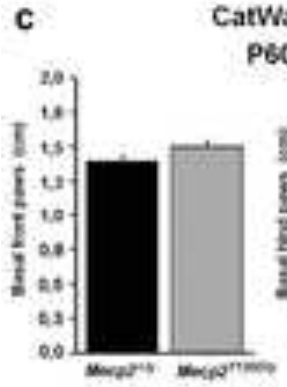

f b

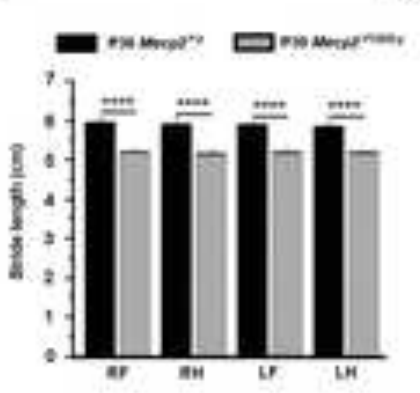

d
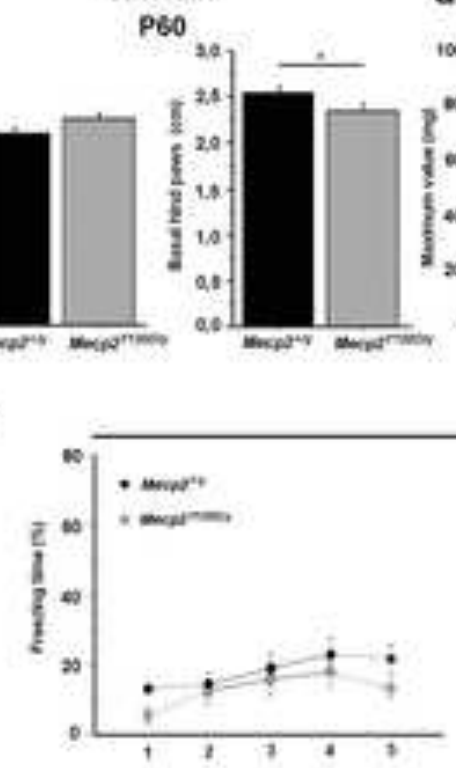

g

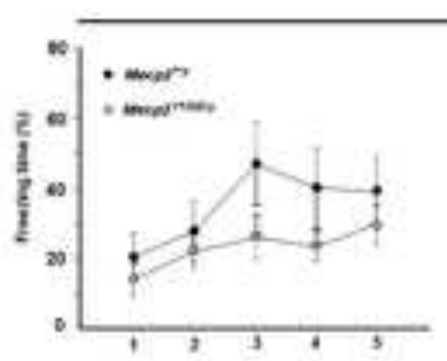

$\mathbf{g}^{\prime}$
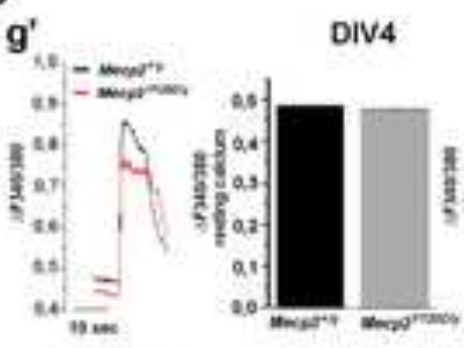

${ }^{h}$ '

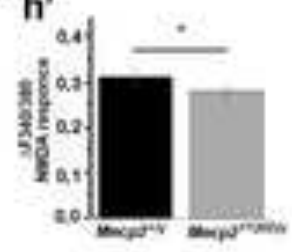

CatWalk

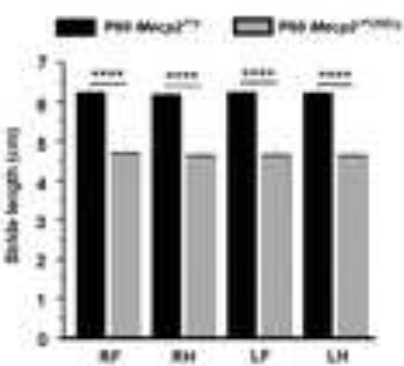

e Spontaneous Altemation

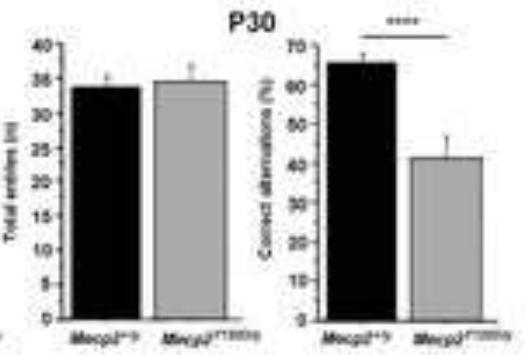

Trace fear conditioning Pyo

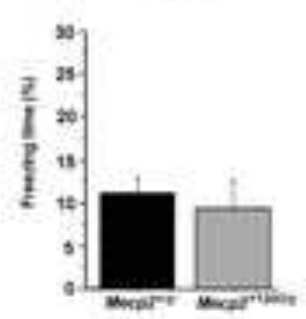

PEO
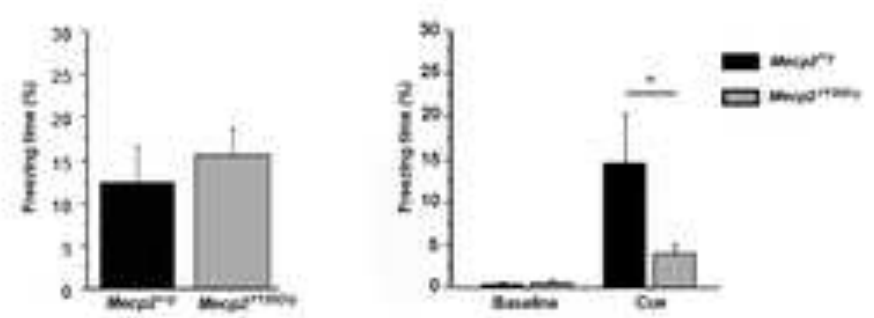

Div13

9"
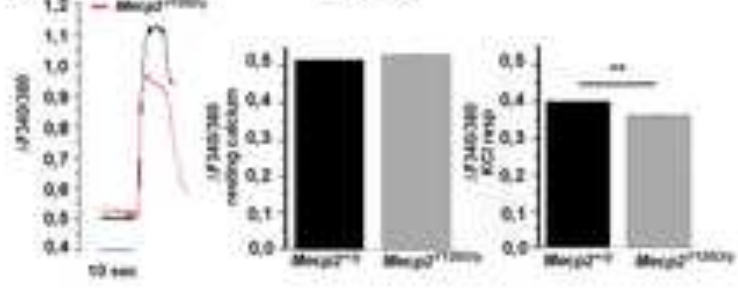

$h^{*}$

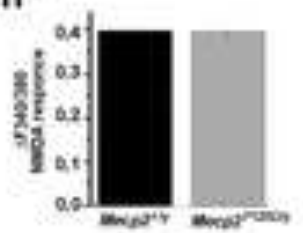


a

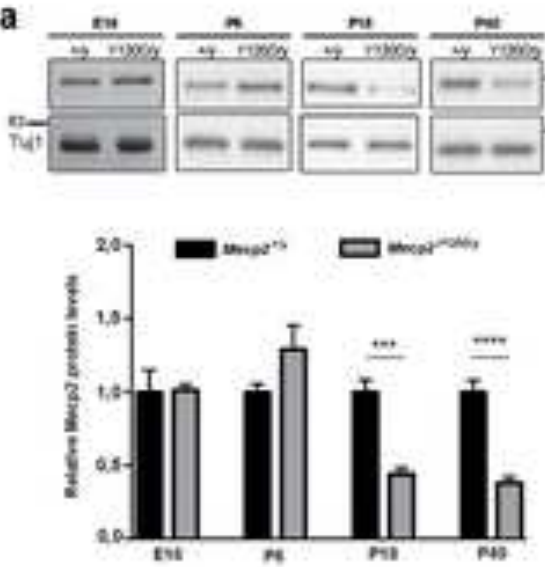

b
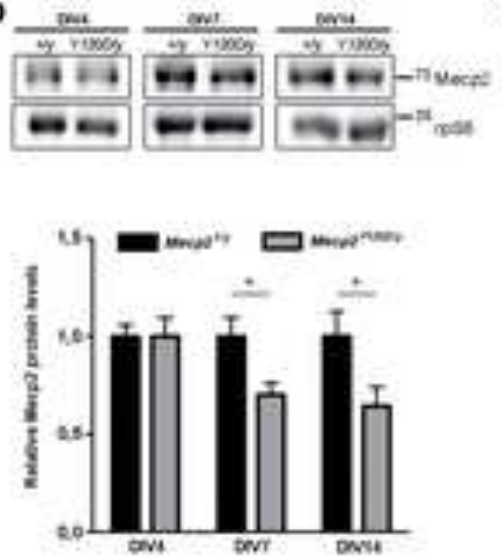

c
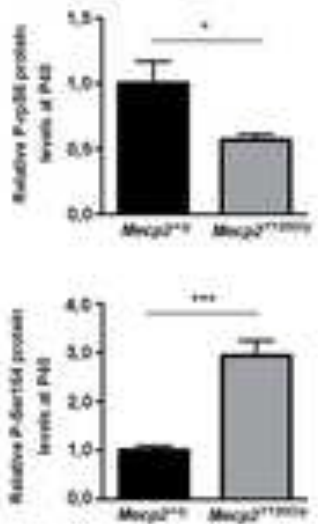

d Mecp 2
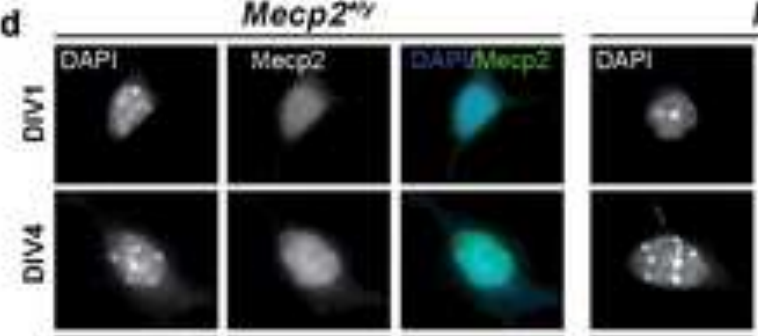

Mecp2 $2^{\text {rasos }}$

5
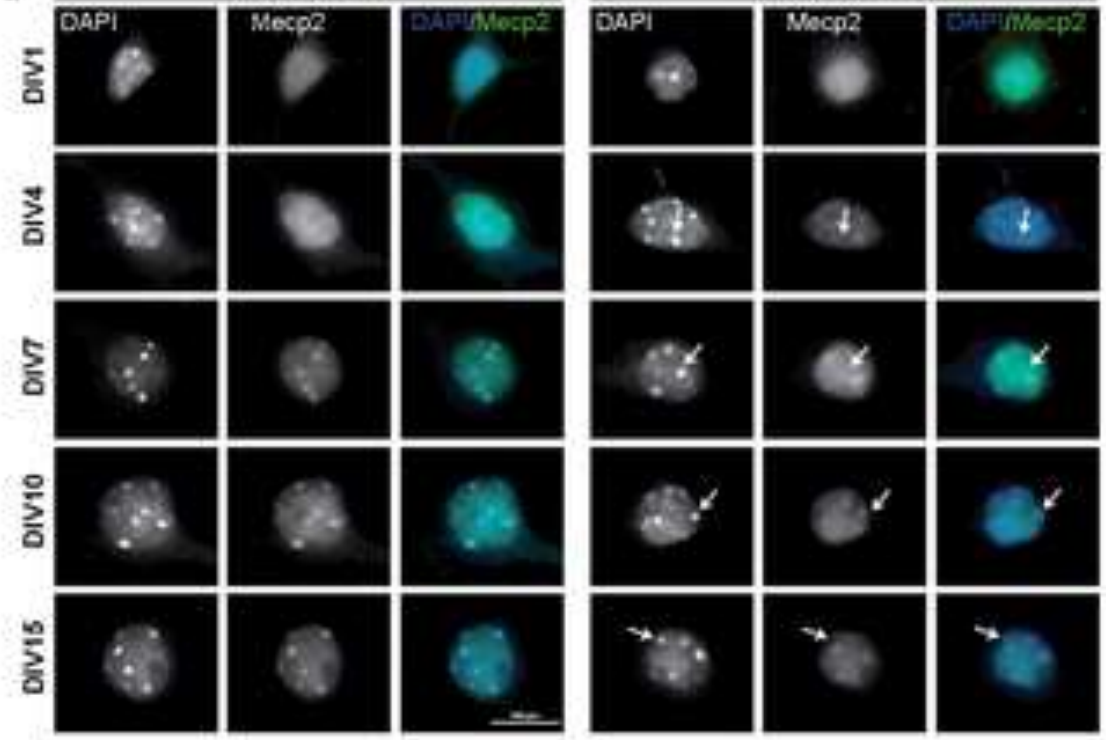

e
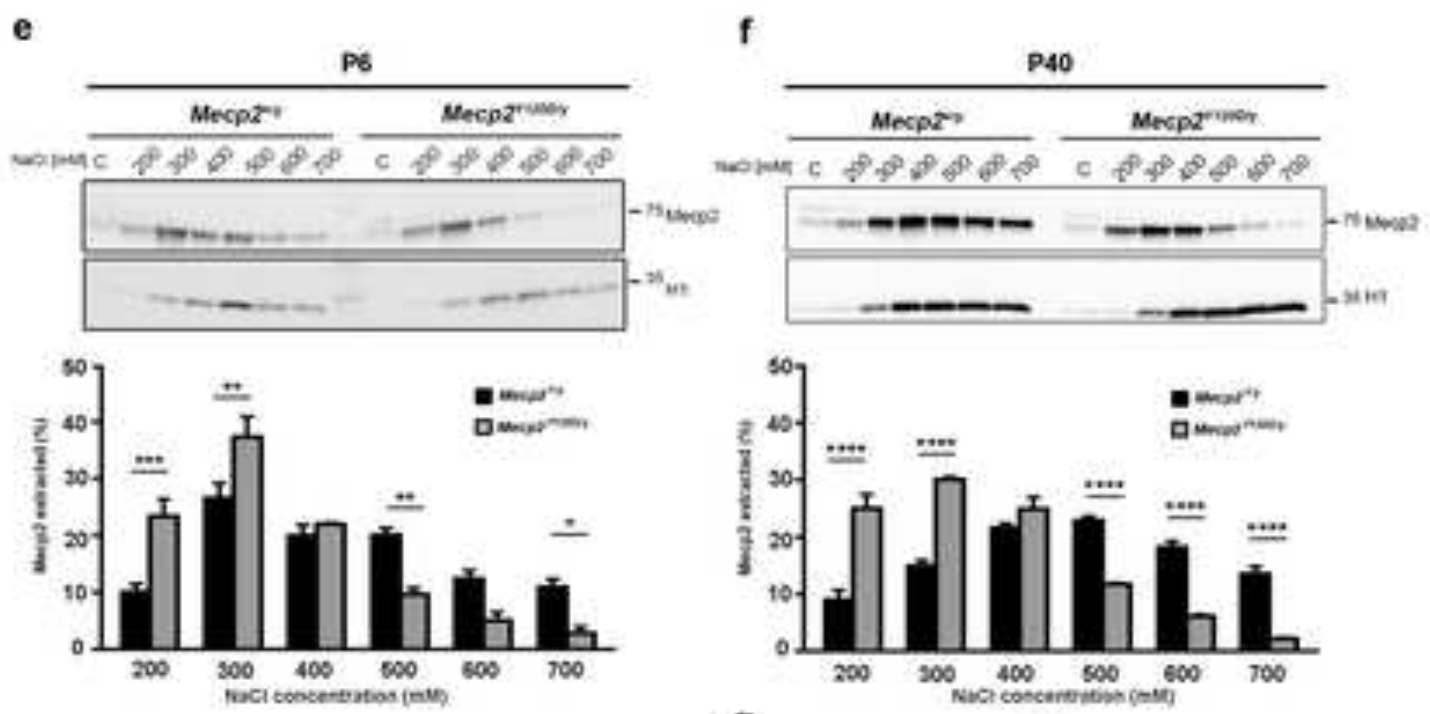

g

P40

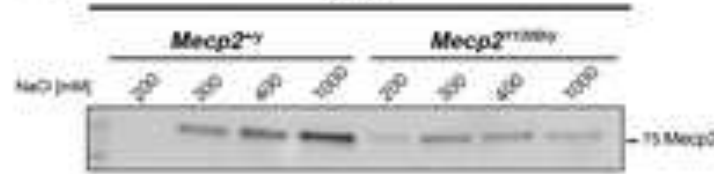




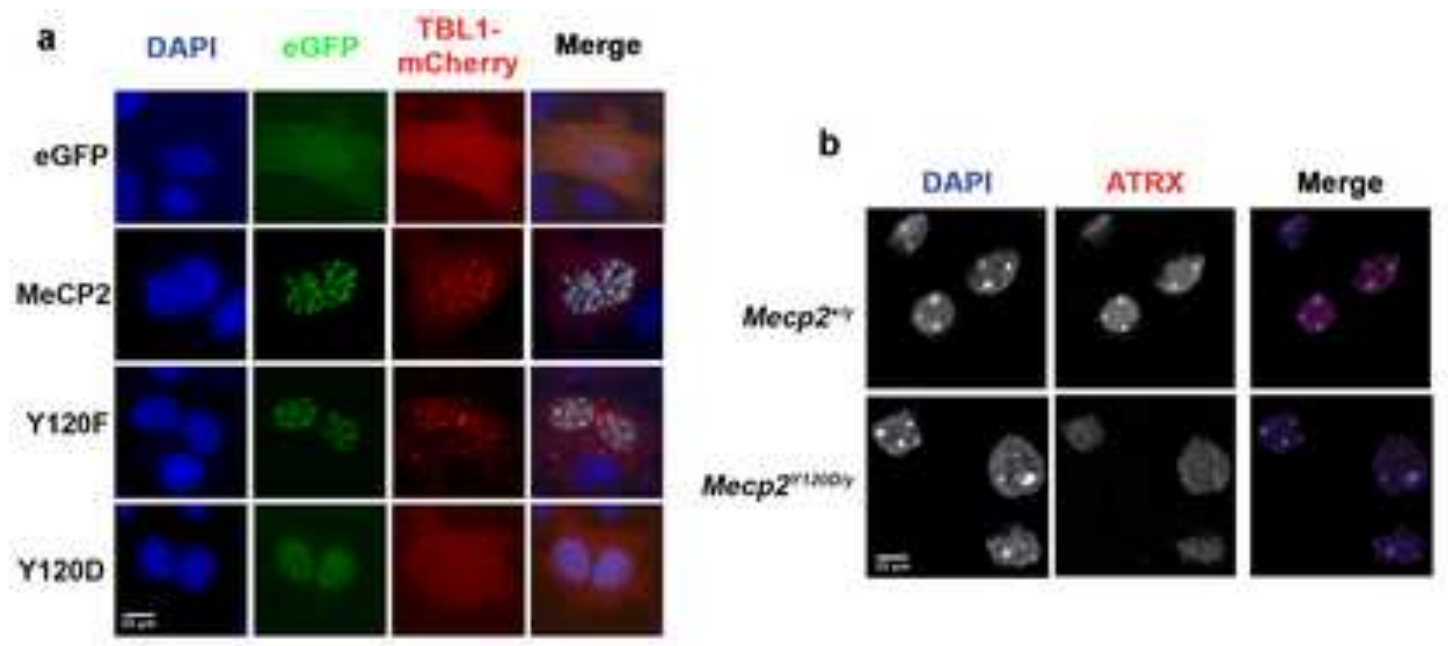


a

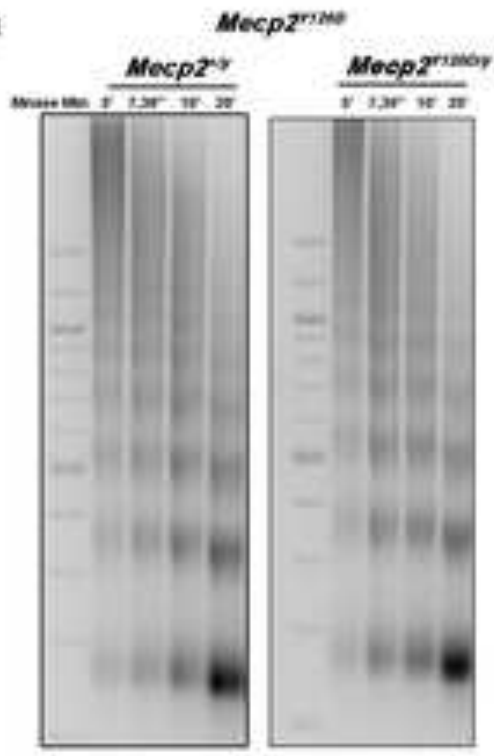

c

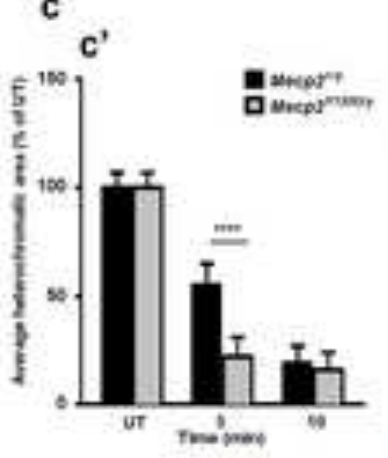

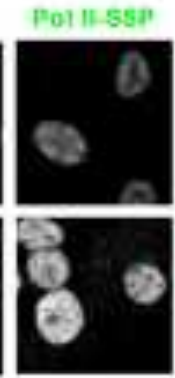

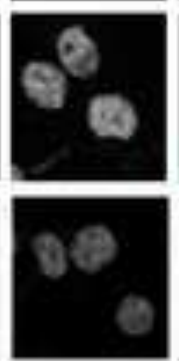

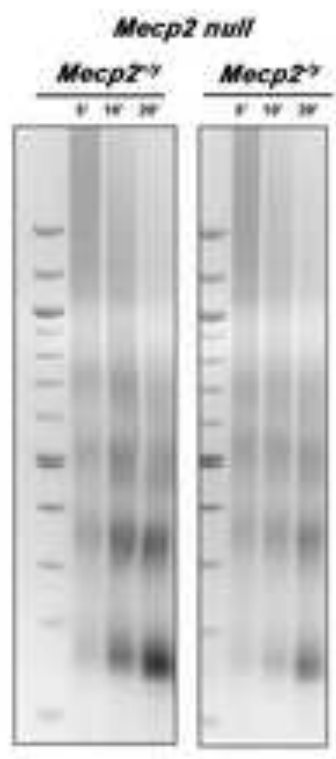

b b
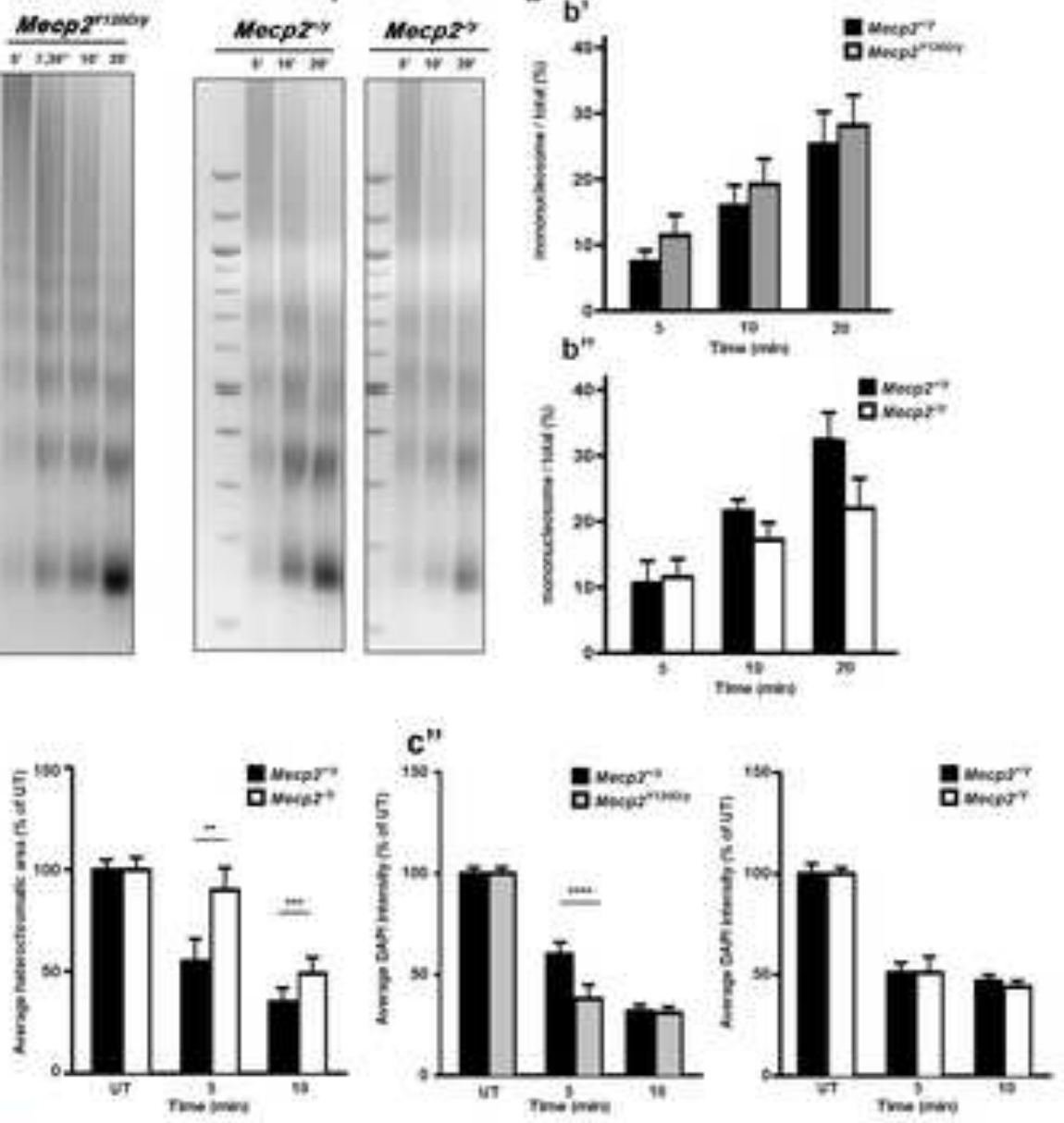

$d^{\prime}$
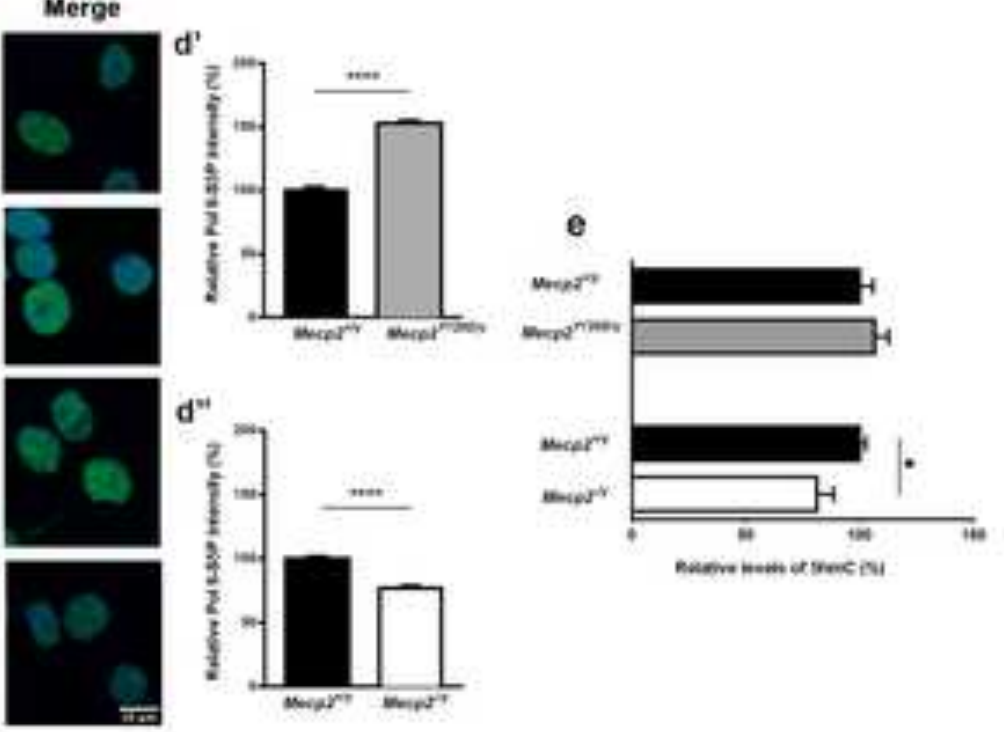
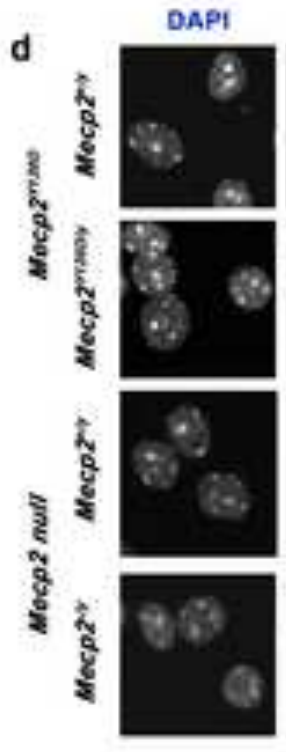
Click here to access/download;Figure;Figure 6.tif $\underline{\underline{\imath}}$

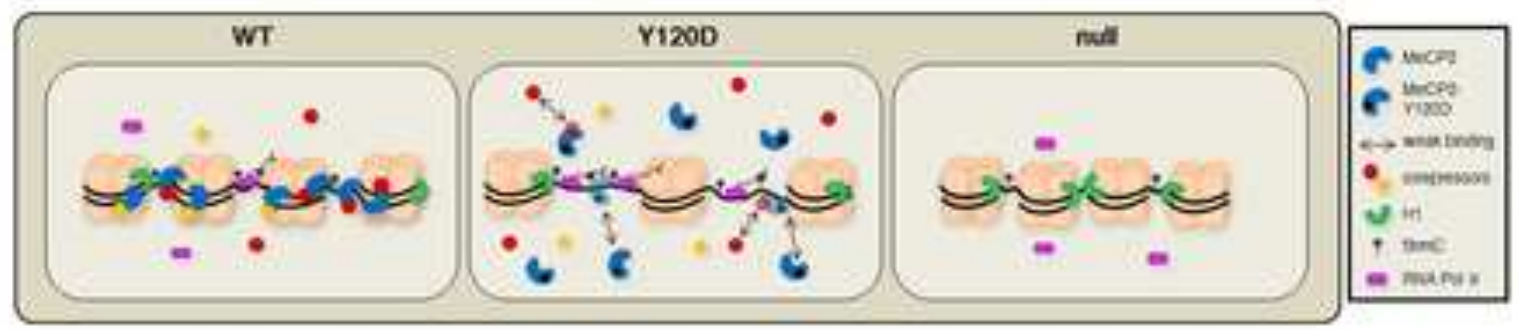

